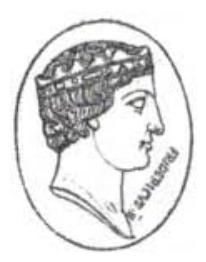

\title{
Ueber die Goldprägungen Kaiser Friedrichs II. für das Königreich Sicilien und besonders über seine Augustalen.
}

Von

\section{E. Winkelmann.}

Einleitung. - 1. Die Einführung der Augustalen. - 2. Beschreibung derselben. Das Bild des Kaisers. - 3. Die Stempel der Augustalen. - 4. Ihr Gewicht. - 5. Ihr Goldgehalt. -6 . Die balben Augustalen. - 7. Ausprägungen nach 1231. - 8. Der Tarenus auri; Vorgeschichte.- 9. Die Tari Friedrichs II. - 10. Gewicht und Gehalt der Tari. - 11. Die Regales Karls von Anjou. - 12. Bisherige Werthbestimmungen der Fridericianisehen Goldmünzen. - 13. Hentiger Metallwerth der uncia anri.14. Metallwerth des Tari und der uncia tarenorum. - 15. Verkehrswerth des Tari. - 16. Metallwerth und Verkehrswerth des Augustalis. - 17. Verhältniss der uncia tarenorum und der uncia augustalium, -18 . Prüfung und Bestätigung der bisherigen Ergebnisse. - 19. Schlussbetrachtungen und Tabellen.

Mancherlei ist schon über die Augustalen Kaiser Friedrichs II. geschrieben worden, jene von ihm für sein Königreich Sicilien geschlagenen Goldmünzen, die sich vor gleichzeitigen anderer Länder durch ihre verhältnissmässig hohe künstlerische Ausstattung und ihre erfolgreiche Nachahmung der Antike auszeichnen. Aber abgesehen von einem Aufsatze von L. Blancard in Marseille ${ }^{1}$ ) ist das Meiste - und ich nehme davon nicht aus, was ich selbst vor 30 Jahren in meiner Geschichte Kaiser Friedrichs II. und seiner Reiche I, 382 aus diesem An-

1) L. Blancard, Des monnaies frappées en Sicile au XIII. siécle par les su. zerains de Provence, in der Revue numismatique. Nouv. série. T. IX (1864), $212 \mathrm{ff}$. $294 \mathrm{ff}$. Aber Bl. spricht hier von den Augustalen nur insofern, als er ihrer als Unterlage für seine Ausführungen über die Münzthätigkeit Karls von Anjou bedarf. 
lasse bemerkte - ziemlich unbefriedigend ${ }^{1}$ ) und man wird nicht behaupten können, dass schon ein wirklicher Einblick in das Wesen, sei es dieser Prägung, sei es überhaupt der sicilischen Goldwährung unter jenem Herrscher genommen worden sei. Die Schuld davon mag ja zum Theil daran liegen, dass die Ueberlieferung selbst nur weniges bietet; denn wir haben an chronikalischen Hilfsmitteln im Grunde nichts als eine allerdings gleichzeitige, aber kurze Nachricht über die Einführung der Augustalen bei Richard von S. Germano und von urkundlichen Quellen auch nur fünf Stücke, nämlich

1) eine wohl noch der staufischen Zeit angehörige Zusammenstellung der in den Münzstätten von Brindisi und Messina beobachteten Geschäftspraktiken, von der der erste, hier fast allein in Betracht kommende Theil bei Blancard a. a. 0. p. 225, das Ganze aber bei Winkelmann, Acta imperii I, 766 gedruckt ist - im Folgenden als Münzerordnung citirt 2); und vier Münzverordnungen Karls I. von Anjou;

2) von 1266 Nor. 5 bei Del Giudice, Codice diplomatico I, 197;

3) von 1271 Jan. 24 bei Minieri Riccio, Il regno di Carlo I di Angiò negli anni 1271 e 1272 , p. 8 ,

4) von 1271 Mai 7, daselbst p. 17, endlich eine

5) von 1273 Jan. 21 im Arch. stor. Ital. Ser. 3 T. XXII, 10.

Unter diesen Umständen kann man nur dann hoffen weiter zu kommen, wenn es möglich wird, die Untersuchung auf recht zahlreiche Exemplare der Augustalen selbst zu stätzen, die allerdings trotz ihrer Einziehung durch König Karl I. nicht allzu selten sind, aber doch an keinem Orte sich in solcher Zahl finden, dass mit ihnen allein sich ein gewisser Abschluss erreichen liesse ${ }^{3}$ ). Ich wäre desshalb auch gar nicht im Stande gewesen, an diese Untersuchung heranzutreten,

1) Bianchini, Della storia delle finanze del regno di Napoli. Ed. II. (Palermo 1839), führt öfters irre. Die Schrift von Valeriani, Ricerche critiche sull'Agostaro di Federigo II. (Bologna 1819) war mir auch jetrt nicht zugänglich.

2) In meiner Ausgabe hatte ich für sie angiovinischen Ursprung vermuthet, wegen mancher Uebereinstimmung mit einer Verordnung Karls I. von 1273. Aber diese Uebereinstimmung beweist nichts, da Karl damals noch sein Münzsystem ganz dem Friedrichs II. angeschlossen hatte, so dass nichts hindert, entweder für jene Ordnung geradezu staufischen Ursprung anzunehmen oder sie, auch wenn sie angiovinisch, d. h. aus der Zeit vor der wirklichen Einführung der Regalen, sein sollte, trotzdem unbedenklich für die Verhältnisse der staufischen Zeit zu verwerthen.

s) Die meisten scheint Neapel zu haben: nämlich 7 ganze und 4 halbe $A u$ gustalen, s. Catalogo del Museo nazionale di Napoli. Medagliere III, 1 nr. 1127 bis 1137. 
wenn nicht meiner Bitte um Mitteilung von Abdrücken und genauen Gewichtsangaben von ganzen und halben Augustalen und den ebenfalls unter Friedrich II. wie seinen Vorgängern und Nachfolgern üblichen Goldtareni überall mit der grössten Bereitwilligkeit entsprochen worden wäre, ausser von Petersburg, von wo ich trotz weitgehender Verheissungen in 13etreff des dort vorhandenen anscheinend sehr reichen Materials schliesslich doch nichts erhielt. Es ist daher nur eine einfache Pflicht der Dankbarkeit, wenn ich derjenigen gedenke, die sich mir in jener Beziehung hilfreich erwiesen: der Herren Beamten des k. Münzkabinets zu Berlin, Dr. Toeche ebendort, Archivrath Dr. Baumann in Donaueschingen (fürstlich Fürstenbergische Sammlnngen), Dr. Davidsohn in Florenz (betr. das Museo archeologico daselbst), Direktor Dr. Pertsch in Gotha (Friedenstein'sche Sammlungen), H. Grueber in London (Brit. Museum), Dr. Riggauer in München (k. Münzkabinet), Museumsdirektor Comm. de Petra in Neapel (Musei di antichità), Direktor Dr. Essenwein in Nürnberg (Germ. Museum), Museumsdirektor Comm. Salinas in Palermo (Nuseo nationale), H. Prou in Paris (Bibl. nation.), Senator Baron Carutti di Cantogno in Turin (R. Medagliere) ${ }^{1)}$ und Dr. v. Schlosser in Wien (k. k. kunsthist. Sammlungen). Auch meinen Kollegen. den Herren Geheimrath Dr. Knies und Professor Dr. Leser, bin ich für mancherlei Auskunft über mir ferner liegende Verhältnisse verpflichtet und ebenso meinen Kollegen H. Professor Dr. v. Duhn, der ausserdem wiederholt seine wirksame Vermittlung an auswärtigen Stellen eintreten liess. Herr Dr. Fester, Docent in München, hatte die Freundlichkeit, mich öfters durch Nachschlagen in der dortigen Bibliothek zu unterstützen.

So konnte ich denn, abgesehen von einem Augustalis im Originale, der als Gabe eines Freundes zur Förderung dieser Untersuchung in meinen Besitz gekommen ist, über Abdrücke und Gewichtsangaben von 36 ganzen, 15 halben Augustalen und 11 Goldtarenen verfügen, wohl über mehr, als irgend jemand vorher beisammen gehabt hat. Dazu kamen die Abbildungen von Augustalen bei Huillard-Bréholles, Recherches sur les monuments des Normands etc. dans l'Italie meridionale pl. XXXI, dann auf dem Titelblatte von desselben Historia diplomatica Friderici II. T. I und bei Salazzaro, Monumenti T. II. tav. $4^{2}$ ).

1) Das königliche Münzkabinet daselbst hat jedoch von Friedrich II. nur eine Silbermünze. Auch der Sammlung in Florenz fehlen Augustalen und ebenso soll es bei der des Vaticans sein.

2) Hier etwas zu gross gerathen. Die Abbildung in Ambrosoli's Numismatjca (Milano 1891) p. 140 ist ganz undeutlich. 
Das waren die Hifsmittel, die mir für die folgende Untersuchung über Friedrichs II. Goldprägungen in Sicilien zu Gebote standen. Es sind von ihr nur die Münzen mit kufischen Inschriften 1) aus naheliegenden Gründen ausgeschlossen worden.

1. Durch die Einführung der Augustalen erlitt das von den Normanen und zum Theil noch aus viel früherer Zeit überkommene Münzsystem des Königreichs Sicilien eine wesentliche Veränderung. Aber sie wird allein von dem gleichzeitigen Annalisten Ryccardus de S. Germano, Mon. Germ. Script. XVIII, 365 berichtet, bei dem es zum December 1231 heisst: Nummi aurei, qui augustales vocantur, de mandato imperatoris in utraque sycla Brundusii et Messane cuduntur. Er erzählt dann p. 368 zum Juni 1232 weiter, dass damals die nova moneta auri, que augustatis dicitur, durch einen kaiserlichen Boten in S. Germano zur Ausgabe gelangte und zwar auf Grund eines kaiserlichen Erlasses, der sub pena personarum et rerum vorschrieb, ut quilibet nummus aureus recipiatur et expendatur pro quarta uncie. Figura augustalis erat habens ab uno latere caput hominis cum media facie et $\mathrm{ab}$ alio aquilam.

Die „distributio" selbst dürfte kaum mehr in derselben Weise geschehen sein, wie die der ersten von Friedrich II. nach seiner Rückkehr ins Könjgreich 1222 geprägten Silberdenare. Damals hatte er es noch in das Belieben der Unterthanen gestellt, sich die neue Münze einzutauschen ${ }^{2}$ ), und dem nur durch das Gebot nachgeholfen, dass eine andere ferner nicht gebraucht werden dürfe ${ }^{3}$ ). Jetzt aber wurde ein anderer, unmittelbarer zum Ziele führender Weg eingeschlagen, wenn wir nämlich den Bericht Ryccards aus der Verordnung Karls I. vom 7. Mai 1271 ergänzen dürfen, die sich ausdrücklich auf den Gebrauch seiner Vorgänger beruft ${ }^{4}$ ). Ein besonderer Beauftragter des Kaisers brachte darnach, ausgerüstet mit einem Mandate desselben, in jeden Stadt- und Landbezirk einen verhältnissmässigen Betrag der neuen Münze, der dort zu dem bestimmten Preise zu übernehmen war. In-

1) Vgl. über diese: Il medagliere Arabo-Siculo, illustrato dal Marchese V. Mortillaro (Palermo 1863) und Amari, Storia dei Muselmani III, $810 \mathrm{ff}$.

9) Winkelmann, Acta imp. I, 763: d. imperator faciebat distribuere monetam ipsam per regnum iuxta voluntatem hominum.

s) Rycc. de S. Germ. Chronica priora ed. Gandenzi p. 108.

4) Im Auszuge bei Minieri, Il regno p. 18: es sollen so viele Mtinzen geschlagen werden, quanta posse essere sufficiente per distribuirsene in tutte le città e terre del reame, come fu praticato da' loro predecessori. 
nerhalb der Gemeinde wird dann der Gesammtbetrag in ähnlicher Weise auf die Einzelnen umgelegt worden sein, wie es mit der Reichssteuer, der collecta, zu geschehen pflegte.

Mehr weiss man über die Einführung der Augustalen nicht und es bleibt dunkel, ob sie glatt oder mit Schwierigkeiten vor sich ging. Dass solche befürchtet wurden, scheint die Drohung mit der pena personarum et rerum anzudeuten.

2. Die Beschreibnng, die Rycc. de S. Germ. von der neuen Münze gibt, ist zwar sehr summarisch, aber an sich nicht unrichtig. Die Augustalen im Durchmesser von 2 Centimetern zeigen, und von den halben Augustalen, deren Rycc. gar nicht gedenkt, gilt das Gleiche, auf der einen Seite einen natürlichen Adler mit geöffneten Flügeln, dessen Kopf nach rechts (vom Beschauer) gewendet ist, und mit der von einem Perlkranze umfassten und durch die Füsse des Adlers getheilten Umschrift + FRIDE | RICVS. Auf der anderen Seite findet sich die ebenfalls nach rechts gekehrte Büste des Münzherrn, der mit einem auf der rechten Schulter durch einen Ring zusammengehaltenen Pallium so beklejdet ist, dass der Hals und der mit Reifen geschmückte rechte Arm frei bleiben; der Kopf trägt einen Blätterkranz, den im Nacken eine flatternde Schleife schliesst; die Umschrift, ebenfalls getheilt und ebenfalls von einem geperlten Kreise umfasst, lautet hier IMPROM | CESARAVG ${ }^{1}$ ).

In diesen Bestandtheilen der Darstellung stimmen alle mir zugänglich gewesenen Stücke üherein, bis auf eins in Wien, das auch darin eigenartig ist, dass es nur einen Durchmesser von 1,75 Centimetern hat, also, da sein Gewicht dem Durchschnittsgewichte der übrigen ziemlich gleich ist, wohl etwas dicker als diese geprägt worden ist. Nur auf diesem einen Exemplare ist der Kopf des Adlers nach links (vom Beschauer) gekehrt, auch in Fridericus das unciale $\epsilon$ verwendet und der übrigens ziemlich kräftig gearbeitete Kopf des Kaisers, statt mit einem Kranze, mit einem Zackendiadem geschmückt.

Es kann beiläufig zweifelhaft sein, welche Seite als Haupt- oder Vorderseite zu gelten hat. Wenn von der Zeit des Rycc. an, wie es scheint, stets die Kopfseite als die vordere angesehen wurde, so möchte dem doch entgegenstehen, dass erst die Adlerseite den Namen des Münzherrn bringt. Kopf und Namen, sonst auf einer Seite vereinigt, sind hier in ganz ungewöhnlicher Weise auf beide Seiten vertheilt worden. Dass die Zeitgenossen dies als etwas ungehöriges empfanden, ist daraus zu schliessen, dass Karl von Anjou, als er die Augustalen

1) Man beachte den Wechsel von $E$ und $\epsilon$ in beiden Umschriften. 
durch seine Regalen ersetzte, wieder in die hergebrachte Weise einlenkte. Weil aber der Namen des Münzherrn erst die eigentliche Gewähr für den Ursprung der Münze gibt und weil das dem Namen vorangesetzte Kreuz anzeigt, dass die Umschrift mit dem Namen beginnt, behandle ich im Folgendeu die Adlerseite als Vorderseite der Augustalen, obwohl es im Grunde gleichgiltig ist, wie man sich entscheidet.

Die Augustalen sind anziehend durch das in der Darstellung des Adlers und des Kopfs des Kaisers sich aussprechende Streben nach Naturwahrheit und durch die damit zusammenhängende, ebenso unverkennbare Anlehnung an die Antike. Woher aber die letztere? Mir scheint die neue Prägung in engster Verbindung mit der ihr unmittelbar rorangegangenen Gesetzgebung Friedrichs II., den berühmten Constitutionen von Melf, zu stehen, die im August 1231 veröffentlicht und selbst auch Augustales genannt1) wurden. Wie sich in diesen Constitutionen das sehr bestimmte Bewusstsein des Kaisers ausspricht, als solcher der Nachfolger der Cäsaren und die Quelle des Rechts zu sein, obwohl das Gesetzbuch nur für das Königreich Sicilien Giltigkeit hatte, so erscheint er auch auf den Münzen, die ebenfalls nur auf das Königreich berechnet waren und ausserhalb desselben im Imperium keinen Kurs haben sollten und, soviel mir bekannt ist, auch nicht gehabt haben, nicht etwa als Kaiser und König von Sicilien, sondern schlechtweg nur als Imperator Romanorum Cesar Augustus, so wenig das auch zu den zwischen ihm und dem Papstthume bestehenden Abmachungen über das Verhältniss des Königreichs zum Kaiserreiche stimmen mochte. Die Sprache der Constitutionen, die Ausstattung der Augustalen und die Benennung beider, sind aus einer und derselben Wurzel entsprungen, eben aus der von Friedrich II. aufs Lebhafteste erfassten Idee des Kaiserthums. Die Stempelschneider der letzteren haben sich demgemäss, und sicherlich nicht ohne Wissen und Willen des Kaisers ${ }^{2}$ ), um ihn darzustellen, Münzen der römischen Kaiserzeit zum Vorbilde genommen, wenn ich auch in der Münzkunde dieser Zeit zu unbewandert bin, um mit Bestimmtheit sagen zu können, gerade welche. Wenn aber für die Gestaltung der Kopfseite der Augustalen vielleicht Kaisermünzen des ausgehenden dritten Jahrhunderts, insbesondere des Probus oder des Diocletian ${ }^{3}$ ), als Vorlage gedient

1) Rycc. d. S. Germ. zu 1232 Febr.

2) Der Kaiser schreibt 1238, als neue Denare geschlagen werden sollten: Sub quibus imaginibus hec nova pecunia cudi debeat, Henricus de Morra, magne curie noster magister iusticiarius, plene per curiam nostram venit instructus.

8) Vgl. Collection de M. le Vicomte de Ponton d'Amécourt nr. 580 ff, $614 \mathrm{ff}$. 
haben möchten, rücksichtlich des Adlers weiss ich eine solche anch nicht einmal zu vermuthen.

Denn auf römischen Münzen erscheint der Adler nicht, wohl aber, worauf mich mein Kollege $\nabla$. Duhn rerwies, sehr häufig auf denen der Ptolemaeer, und da diese sich nach seiner Mittheilung zahlreich in Unteritalien und Sicilien finden, wäre an sich die Möglichkeit nicht abzustreiten, dass sie die Aufnahme des Adlers in den Stempel der Augustalen veranlasst haben könnten, um so mehr, als der Adler sich zugleich als Symbol der von Friedrich II. stark betonten kaiserlichen Würde empfahl.

Trotzdem kann ich mich nicht tür jene Herleitung des Adlers aus den Münzen der Ptolemaeer erklären. Sie würde nur dann als zweifellos gelten können, wenn der Adler der Augustalen auch nur annähernd dem der Ptolemaeer ähnlich wäre. Das ist aber durchaus nicht der Fall: er ist nicht blos überhaupt anders stilisirt als dieser ${ }^{1}$ ), sondern er ist auch stets von Vorne, nicht wie dieser von der Seite dargestellt. So müssen wir denn wohl vorläufig dabei bleiben, dass der Adler seine Aufnahme in die Augustalen nicht der direkten Nachahmung einer bestimmten Münze, sondern einem besonderen Befehle des Kaisers and seine vortreffliche Gestaltung der Beobachtungsgabe der kaiserlichen Stempelschneider verdankt, die dadurch ihrer Befähigung ein rühmliches Zeugniss ausgestellt haben. - Uebrigens kommt der Adler mit geöffneten Flügeln auch schon auf einigen sicilischen Silber- und Kupfermünzen Friedrichs vor, die vor seiner Kaiserkrönung, zwischen 1209 und 1220 geschlagen sind ${ }^{2}$ ), und ebenso auf vielen Goldmünzen ron ihm mit kufischen Inschriften, unter diesen sogar paar Mal zweiköpfig ${ }^{3}$ ).

Besondere Aufmerksamkeit wird sich aber natürlich stets auf den Kopf des Kaisers richten und die Frage wird aufgeworfen werden müssen, ob wir denn hier wirklich die Züge des grossen Fürsten vor uns haben oder ob wenigstens eine annähernde Aehnlichkeit vou den Stempelschneidern erreicht ist. Böhmer meinte in der Einleitung zu seinen Regesta imperii 1198-1254 p. XXXV: „Das Brustbild, welches sich auf seinen goldenen Augustalen findet, ist allerdings gleichzeitig,

t) Man sehe die schőnen Abbildungen zu dem Catalogue of Greek coins in the British Museum. (VI:) The Ptolemies.

2) Catalogo del Museo Nazionale di Napoli. Medagliere III. Parte I Nr. 1123 bis 1126.

9) Ibid. Nr. 1078. 1079. Ich finde nicht, dass diese merkwürdige Erscheinung bei den bisherigen Erörterungen über den Ursprung des zweiköpfigen Adlers beachtet worden ist. 
aber der Antike nachgebildet, und in einer Zeit, welche das Portrait noch wenig oder gar nicht kaunte, ohnedies von zweifelhaftem Werth". Vielleicht müsste man noch weiter gehen und jene Frage ohne Weiteres verneinen; ich selbst habe sie auch früher verneint, und zwar vor Allem aus dem einfachen Grunde, weil Ryccard de S. Germano, der nicht bloss im Dienste des Kaisers stand, sondern bei dessen öfterem Aufenthalte in S. Germano ihn auch häufig genug gesehen haben muss, unmöglich von dem Bilde auf den Augustalen den befremdenden Ausdruck "caput hominis" gebraucht haben könnte, wenn er in demselben die Züge seines Herrn wiedererkannt hätte. Dazu kommt ein Zweites. Der arabische Geschichtsschreiber Yafei sagt nach dem Berichte eines Schaffners der Moschee Omars, der den Kaiser 1229 in Jerusalem begleitet hatte, dass dieser kahl gewesen sei ${ }^{1}$ ); auf den Augustalen aber erscheint er zwar bartlos, jedoch mit reichem Haupthaar, so dass wenigstens in dieser Beziehung hier, wenn auch nicht eine Idealisirung, so doch eine gewisse Verschönerung stattgefunden hätte, wie solche in gleichem Falle wohl auch in neuerer Zeit für zulässig gehalten wird, die aber immerhin die Aehnlichkeit zu beeinträchtigen geeignet ist. Indessen ist es fraglich, inwiefern jene Angabe des Mohammedaners überhaupt Glauben verdient, der, indem er (und ebenso Hassan ibn Ibrahim ${ }^{2}$ ) den Kaiser auch noch roth und kurzsichtig nennt, ihn offenbar als einen äusserlich unbedeutenden Menschen hinstellen will. Nun ist das zwar auch die Ansicht des Ricobaldus Ferrariensis ${ }^{3}$ ): Fuit Fr. non procerus, obeso corpore, subrufus, und wohI nach ihm auch die des Benvenutus von Imola, bei dem es von Friedrich heisst: fuit stature communis, facie letus, colore subrufus, habens membra quadra. Aber Ricobald schrieb erst am Ende des Jahrhunderts und Benvenutus ist durch hundert Jahre von Friedrich getrennt, beide können also nicht gegen den Minoriten Salimbene aufkommen, der den Kaiser 1238 sah und mit folgenden Worten schildert: Pulcher homo et bene formatus, sed medie stature fuit. So bleibt am Ende von jenen ungünstigen Urtheilen über seine äussere Erscheinung nichts übrig, als dass er nur von mittlerem Wuchse, vielleicht anch roth (blond) war, worauf es aber hier nicht weiter ankommt. Die Kahlköpfigkeit, wenn sie vorhanden war, müsste Salimbene aufgefallen sein.

Während der Ausdruck des Annalisten von S. Germano dazu zu

1) Böhmer p. XXVIIl.

2) Michaud VI, 810.

s) Muratori, Script. IX, 132. 
nöthigen scheint, jede Aehnlichkeit des Bìldes der Augustalen mit dem wirklichen Aussehen des Kaisers zu läugnen, gibt es doch auch einige Anhaltspunkte zur Behauptung des Gegentheils 1).

Den ersten Anhaltspunkt bietet der Umstand, dass alle für die Augustalen gebrauchten Stempel unverkennbar dieselben Züge zeigen, nur mit den Abweichungen, die sich aus der subjektiven Auffassnng und dem verschiedenen Grade der Befähigung der einzelnen Stempelschneider ergeben, so dass sie z. B. bald jugendlicher und gerundeter, bald schärfer und magerer erscheinen.

Zweitens kommt eine einst im Besitze Danieles, dann in dem Fr. von Baumers 2) befindliche Gemme in Betracht, die in Baumers Hohenstaufen Bd. III am Schlusse des sechsten Buchs und anscheiuend getreuer auf dem Titelblatte von Huillards Hist. dipl. T. I, und darnach oben auf Seite 401 abgebildet ist. Daniele hatte sie im vorigen Jahrhundert nach dem damals noch vorhandenen Kopfe jener Bildsäule desKaisers herstellen lassen, die dieser an dem von ihm 1.234 nach seinen eigenen Plänen begonnenen Brückenthore von Capua nebst den Bildsäulen des Petrus de Vinea und des Thaddeus de Suessa angebracht hatte ${ }^{3}$ ). Je länger ich nun die Abbildung dieser vielleicht etwas idealisirenden Gemme mit dem Kopfe des bei Huillard daneben gestellten Augustalis vergleiche, um so mehr drängt sich mir jetzt die Verwandtschaft zwischen ihnen auf und ich denke, es wird auch anderen so gehen. Von untergeordneter, aber doch nicht ganz zu unterschätzender Bedeutung ist es, dass der Kaiser auch auf der Gemme das Zackendiadem trägt, das ihm der Stempelschneider des Wiener Augustalis gegeben hat.

Drittens sind auch die Siegel Friedrichs heranzuziehen. Zwar hat die Vergleichung eines Profilbildes, wie Münzen es zeigen, mit der auf den Sigeln allein vorkommenden Vorderansicht immer etwas missliches, und ich bin deshalb weit davon entfernt, darauf Gewicht zu legen, dass mir persönlich die Züge des Siegelbilds mit denen des Münzbilds übereinzustimmen scheinen. Grösseres Gewicht lege ich dagegen darauf,

1) Das Medaillon an der Façade der Kirche von Andria, das Huillard angezogen hat (vergl. die Abbildung Huillard, Recherches pl. XXVIII. XXIX), kann gar nicht in Betracht kommen. Denn es ist ganz ungewiss, wen es darstellen soll, und die Façade selbst rührt erst aus der Renaissance her. H. W. Schulz, Denkmäler der Kunst Unteritaliens I, 150.

2) Vgl. Raumer, Hohenstaufen (1. Ausg.) Bd. III. Vorrede S. VIII. Wo sie jetzt ist, weiss ich nicht.

sj Rycc. de S. Germ. p. 372 a. 1234 (c. April) vgl. B.-F. nr. 20412. Den Kopf der Statue sollen erst die Soldaten Murats abgeschlagen haben. Vergl. Schulz, Denkmäler II, 167. Salazzaro, Monum. I, 55. Huillard-Bréholles, Hist. dipl, Introduct. p. 549 not. 
dass überall, anf den Augustalen, auf der Gemme und auf den Siegeln, der Kaiser völlig bartlos ist: es kann kein Zweifel sein, dass man sich wenigstens in dieser Beziehung an die Wirklichkeit gehalten hat.

Ist es nun denkbar, dass die Verfertiger der verschiedenen Stempel zu den Münzen nicht blos sammt und sonders zufällig auf dieselben Züge verfielen, sondern sich ebenso zufällig darin auch mit dem, doch wahrscheinlich nicht in ihrer Mitte zu suchenden Urheber der Bildsäule von Capua begegneten? Da liegt es doch näher, dass sie alle einem und demselben Vorbilde folgten. Und wenn dem so ist, kann dieses Vorbild ein anderes gewesen sein als die wirkliche Persönlichkeit des Kaisers? Man mag bereitwillig einräumen, dass einige ihr nicht ganz gerecht geworden seien - auf manchen Stempeln ist der Kopf von einer gewissen Härte - , oder dass andere sie mehr oder minder idealisirt haben, aber so weit möchte ich darum doch nicht gehen, dem Produkte ihrer künstlerischen Thätigkeit alle und jede Naturwahrheit abzustreiten. Vielmehr scheint mir daran festgehalten werden zu müssen, dass bei den Augustalen Portraitähnlichkeit beabsichtigt und von den im Durchschnitte befähigten Stempelschneidern auch einiger Massen erreicht worden ist. Allerdings muss ich aber auch bekennen, dass es mir bei dieser Auffassung ein psychologisches Räthsel bleibt, wie Rycc. de S. Germ. auf seinen in jedem Falle sonderbaren Ausdruck "caput hominis" gekommen sein mag.

3. Dass nicht nur ein Stempel sondern mehrere, unter Festhaltung des allgemeinen Typus, für die Ausprägung der Augustalen zur Verwendung kamen, ist natürlich und wird durch eine genauere Betrachtung der einzelnen Prägestücke bestätigt, die vielfach kleine Abweichungen von einander aufweisen: in der Gestaltung des Adlers und besonders seiner Flügel, in den Linien des Kaiserbildes, in der Grösse und Sperrung der Buchstaben und in der Verwendung von Punkten und Punktkreisen in den Umschriften. Nach diesen Merkmalen und namentlich nach den Interpunktionen, die leichter fassbar sind als die anderen, habe ich die für die Augustalen gebrauchten Stempel zu sondern versucht und im Folgenden aufgezählt. Es ergiebt sich dabei u. a., dass häufig bei den Prägungen Vorder- und Rückseite mit Stempeln geschlagen wurden, die ursprünglich nicht zusammengehörten, so dass dadurch eine grosse Maunigfaltigkeit in der äusseren Erscheinung der Münze zu Stande kam. Das aber ist selbstverständlich, dass mit dieser Aufzählung die Zahl der wirklich gebrauchten Stempel schwerlich erschöpft sein dürfte, und andrerseits bin ich selbst keinerwegs davon überzeugt, dass in der Zutheilung der 
einzelnen Augustalen an diesen oder jenen Stempel nicht hie und da auch ein Versehen untergelaufen ist. Indessen man wird in dieser Beziehung, wenn es wirklich der Fall sein sollte, wohl Nachsicht walten lassen, in der Erwägung, dass jene Scheidung nicht das Ergebniss unmittelbarer Vergleichung der Originalmünzen ist und sein konnte, sondern sich nur auf Abdrücke stützt, die nicht immer deutlich waren und auch sonst oft viel zu wünschen übrig liessen oder beschädigt an mich gelangten 1). Wo mir aus einem Kabinette mit den Abdrücken auch die Nummern der Stücke mitgetheilt wurden, habe ich diese angeführt, wo das aber nicht geschah, und das war bei der Mehrzahl der Fall, die einzelnen Stücke zur Unterscheidung selbst nummerirt, bei jedem aber auch in Klammern gleich sein Gewicht angemerkt ${ }^{2}$ ). Da diese Angaben jedoch durchaus nicht auf eigenen Wägungen fussen, muss ich die Verantwortung für dieselben ablehnen, habe aber allerdings keinen Grund, ihre Genauigkeit zu bezweifeln.

Ich glaube also, bei den mir bekannt gewordenen Augustalen folgende Stempel unterscheiden zu können, indem ich nur noch darauf hinweise, dass, wenn rücksichtlich einzelner Elemente der Darstellung nichts bemerkt ist, von diesen das oben von den Augustalen überhaupt Gesagte Geltung hat:

I. Vorderseite: Adler, nach rechts vom Beschauer gekehrt. Umschrift: + FRIDE | RICVS (ohne Punkt).

Rückseite: Büste des Kaisers mit Blätterkranz, rechts gekehrt. Umschrift: 'IMPROM- | CESARAVG'

München $3(5,30)$, Paris Nr. $999(5,28)$, Wien 4 (5.25).

II. $V$.: wie in I, aber das schliessende $S$ etwas geneigt. R.: AVG gesperrt.

Gotha $(5,26)$, Wien $1(5,25)$. - Nach letzterem Nr. 1 der Tafel.

1) Das trifft leider besonders die aus dem Museum zu Neapel, von dessen 7 Augustalen (die meisten, die eine Sanmlung zu haben scheint) überdies mir nur sechs in Abdrücken zur Verfügung standen.

3) Letzteres konnte bei den Augustalen in Neapel nicht geschehen, weil mir von dort her zwar sehr genaue Gewichtsangaben sämmtlicher Stücke mitgetheilt wurden, aber nicht, auf welchen Abdruck sich die einzelne Angabe bezog. Jene mögen deshalb nach den Museumsnummern hier besonders aufgeftubrt werden.

\begin{tabular}{|c|c|c|}
\hline Nr. 1127 & Gr. 5,263 & \\
\hline Nr. 1128 & Gr. 5,263 & Nr. 1131 \\
\hline Nr. 1129 & Gr. 5,260 & Nr. 1132 \\
\hline
\end{tabular}

Nr. 1133 Gramm 5,288. 
III. $V .:$ wie II.

$R$. : wie I.

London 1 (5,796 ?), München 2 (5,30), Neapel zwei Exemplare, Palermo $1(5,30)$.

IV. V.: wie II, dazu aber rechts und links rom Kopfe des Adlers über jedem Flügel ein starker Punkt.

$\boldsymbol{R}$.: wie I. In AVG ist das A heruntergezogen.

London $2(5,248)$, Nürnberg $(5,29)$, München $1(5,32)$, Palermo $2(5,30)$, Palermo $3(5,27)$. - Nach letztem Nr. 2 der Tafel.

V. V.: wie I.

$\boldsymbol{R}$. : wie IV.

Berlin $3(5,29)$.

VI. V.: wie II.

$\boldsymbol{R}$. : oIMPROM (ohne Punkt dahinter) | CESARAUG (ohne Punkt) In Cesar ist $\mathrm{AR}$ gesperrt.

Berlin $4(4,28)$, Donaueschingen $1(5,28)$, Donaueschingen $2(5,25)$, Neapel vier Exemplare, Paris Nr. $1000(5,24)$, Toeche in Berlin $(4,936)$, Winkelmann (5,265). - Vgl. Nr. 3 der Tafel nach Berlin 4 und Nr. 4 nach Donaueschingen 2.

VII. V.: wie II.

$R$. : wie VI, doch mit Punkt hinter ROM.

München $4(5,30)$.

VIII. $V$. : wie I.

R.: oIMPROM (ohne Punkte) | oCESARAVG (ohne Punkt).

Berlin $2(5,28)$.

IX. V.: wie IV.

R.: 'IMPROM (ohne Punkt)|'CESARAOGG (ohne Punkt). SAR etwas gesperrt.

Berlin $1(5,30)$, Wien $2(5,25)$. - Abbildungen bei Huillard-Brèholles und Salazzoro, bei letzterem zu gross, s. o. S. 403.

X. V.: sonst wie $\mathrm{I}$, doch ist das $\mathrm{C}$ unverhältnissmässig gross. Ueber dem rechten Flügel des Adlers ein schwacher Punkt.

R.: wie IV.

Wien $3(5,25)$.

XI. V.: ein I, doch $\mathrm{C}$ grösser.

$R$. oIMPROM - 'CESARAUG. - G zusammengedrückt.

Wien $5(5,25)$,

XII. V.: wie II.

R.: 'IMPROM (ohne Punkt | 'CESARAVG' Das S geneigt und heruntergezogen; AVG gesperrt, $\mathrm{C}$ sehr klein.

Wien $6(5,25)$.

XIII. $V .:$ wie II.

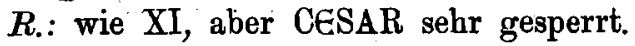

London 3 (5.504), 4 (5,504). 
XIV.: Ganz eigenartig; hat nur 1,75 Centimeter Durchmesser, während die übrigen 2 Centimeter haben.

V.: Der Adler kehrt den Kopf nach links (vom Beschauer) und hat weiter geöffnete Flügel als sonst. Zwischen dem Kopfe und dem linken Flügel des Adlers ein starker Punkt. Umschrift kleiner als sonst:

\section{+ FRIDE | RICVS.}

Der Schlusspunkt und das unciale $E$ an dieser Stelle kommen nur bei diesem Stempel vor.

$R_{\text {:: }}$ Der wie gewöhnlich nach rechts (vom Beschauer) gekehrte Kopf des Kaisers ist höher herausgearbeitet als sonst und trägt ein Zackendiadem, daher keine Schleife im Nacken. Die Büste reicht nur bis wenig unter der Schulter. Die Umschrift ist in ihrem zweiten Theile sehr klein und undeutlich: -IMPROM (ohne Punkt) | (ohne Punkt) CESARAVG.

Wien 8 (5,22). - Darnach Nr. 5 der Tafel.

Irre ich nun nicht, so sind bei den 36 Exemplaren von Augustalen, deren Beschreibung ich zu geben vermochte, also 6 Stempel für die Vorder- oder Adlerseite und 11 für die Rück- oder Kopfseite in Anwendung gekommen und es ist vorauszusetzen, dass in Wirklichkeit die Zahl der überhaupt gebrauchten Stempel eine noch grössere gewesen sein wird, wie sich wohl bei Vergleichungen mit noch anderen Exemplaren herausstellen dürfte.

4. Während den Kunsthistoriker vorzugsweise das Gepräge der fridericianischen Goldmünzen interessiren wird, kehrt sich das Interesse des Historikers und des Volkswirthschaftlere mehr den Fragen nach ihrem Gewichte, ihrem Goldgehalte und ihrem aus beiden sich ergebenden Werthe zu.

Zum Verständnisse der folgenden Erörterungen ist vorauszuschicken, dass in Unteritalien und Sicilien wenigstens schon unter den Normannen, dann aber auch unter ihren Nachfolgern überhaupt, auf dein Festlande bis 1818, auf der Insel sogar bis 1864, folgendes Gewichtssystem bestand:

$$
\begin{aligned}
1 \text { Pfund (libra) }=12 \text { Unzen } \\
1 \text { Unze }=30 \text { Tari (tareni) } \\
1 \text { Tari }=20 \text { Gran, }
\end{aligned}
$$

und dass das Münzsystem sich nicht nur in den Namen, sondern auch in Wirklichkeit dem anschloss. Die vor der Einführung der Augustalen und halben Augustalen allein ausgeprägte Goldmünze, der Tarenus auri, war also in der That $1 / 30$ Unze ${ }^{1}$ ), während die Unze

1) Vgl. Faraglia, Storia dei prezzi in Napoli p. 24: L'oncia ad pondus, 
selbst niemals zur Ausprägung gelangte, sondern von Alters nur eine Rechnungsmünze war und solche auch in der staufischen Zeit und weiterhin blieb, ebenso wie der Gran, der schon seiner Kleinheit wegen gar nicht in Gold gemtinzt werden konnte.

Der Augustalis sollte nun nach dem kaiserlichen Einführungsedikte, von dem Rycc. de S. Germ. leider nur einen dürftigen Auszug giebt, ein Viertel Unze ( $=7 \frac{1}{2}$ Tari) gelten und dafür von Jedermann sub pena personaram et rerum angenommen werden. Diese Audrohung hatte ihren gaten Grund, da sich befürchten liess, dass die Privaten sich wenigstens anfänglich gegen die Aufnahme der neuen Mänze sträuben würden ${ }^{1}$ ), deren wirkliches Gewicht - abweichend von dem gewohnten Goldtari - weit hinter dem angenommenen zurückblieb. Es betrug nämlich nicht $7 \frac{1}{2}$, sondern nur 6 Tari, wie wir aus der Verordnung Karls vom 5. Nov. 1266 erfahren, durch die er für den Augustalis, aber mit Beibehaltung aller Verhältnisse desselben, seinen Regalis einführte. Dem entsprechend wog der halbe Augustalis nicht 3 Tari 15 Gran, sondern nur 3 Tari ${ }^{2}$ ).

Wurde nun diese gesetzliche Feststellung des Gewichts auf sechs Tari, die böchst wabrscheinlich auch schon in dem kaiserlichen Einführungsedikte von 1232 gestanden hat, bei der Ausprägung der Augustalen wirklich innegehalten? Um darauf antworten zu können, muss zunächst ermittelt werden, wie viel ein Tari selbst nach heutigem Systeme wog. Wir sind nicht berechtigt, dem heute noch in Sicilien bei Goldschmieden u. a. üblichen Pfunde von 317,37 Gramm und der Unze von $26,45 \mathrm{Gramm}^{3}$ ), wonach ein Tari $=0,88 \mathrm{Gr}$. sein würde, ohne Weiteres auch für das dreizehnte Jahrhundert Giltigkeit beizulegen.

Die 36 Augustalen, von denen ich Gewichtsangaben habe ${ }^{4}$ ),

come si è detto, corrispondeva esattamente all' oncia moneta (solche gab es nicht) percio il tari era la trentesima sua parte, come peso e come valore di moneta.

1) Dass kein wirklicher Grund zur Beunruhigung vorlag, werden wir später sehen. Doch mag schon vorgreifend hier bemerkt werden, dass der Augustalis genau so viel Feingold enthielt als $7 \frac{1}{2}$ Goldtari.

2) Del Giudice, Cod. dipl. del regno di Carlo etc. I, 197: quilibet regalis sit in pondere tarenorum sex et medius regalis tarenorum trium ...., prout augustales et medii augustales olim erant dicte tenute et ponderis.

s) Mittheilung des H. Comm. Ant. Salinas. - Nach Bleibtreu, Münz-, Massund Gewichtskunde S. 305 hatte das alte neapolitanische Pfund $=320,76 \mathrm{Gr}$., das sicilische Handelspfund nach S. 560 dagegen nur 317,552 Gr.

4) Das Exemplar des H. Dr. Toeche mit seinen 4,93 Gr. ist bei diesen Berechnungen deshalb ausgelassen, weil es ersichtlich in ganz ungewöhnlichem Grade minderwerthig geworden ist. 
schwanken in ihrem Gewichte zwischen 5,796 und 5,188 Gr., also um mehr als $1 / 2$ Gr. oder ein Zehntel, während die Schwankungen im Gewichte der deutschen Doppelkronen 21/2 Tausendstel nicht uibersteigen dürfen. Als mittleres Gewicht ergab sich für sie 5,297 Gr. ${ }^{1}$ ). Ist es in einigen Stücken erheblich überschritten, so bleibt doch die Mehrzahl hinter demselben zuriick und man kann wohl annehmen, dass das Plus bei jenen nur auf Zufälligkeiten zurückzuführen ist.

Aber jenes Durchschnittsgewicht ist in der That nicht als das ursprüngliche zu betrachten, mit dem der Augnstalis in der Regel aus der Münzstätte hervorging. Um dies zu erhalten, muss jenem noch etwas zugesetzt werden als Ersatz dessen, was der Münze durch Abnützung u. s. w. verloren gegangen ist. Wenn es zutreffend ist, dass, wie Blancard will, etwa ein Procent von dem heutigen Gewichte auf jenen Abgang zu rechnen ist ${ }^{2}$ ), würde sich das Durchschnittsgewicht der Augustalen um 0,053 Gr., also auf 5,350 Gr. erhöhen und diese den 6 Tari eutsprechen, die sie bei ihrer Ausgabe wiegen sollten.

Hieraus müssten folgende Gleichsetzungen gefolgert werden:

$$
\begin{aligned}
& \text { der Tarenus } \frac{5,350}{6}=0,891 \text { Gr. } \\
& \text { die Unze } 0,891 \times 30=26,730 ", \\
& \text { das Pfund } 26,730 \times 12=320,760 "
\end{aligned}
$$

Dieses Ergebniss kann jedoch anch noch nicht als unbedingt richtig gelten und auch so dürfen wir noch nicht hoffen, das ursprüngliche gesetzliche Gewicht des Augustalis vollständig genau gewonnen zu haben, da einerseits jener Zuschlag mehr oder minder willkürlich ist und andrerseits die Berechnung zwar auf verhältnissmässig zahlreichen Wägungen, aber doch eben nur auf solchen der zufällig zu meiner Kenntniss gekommenen Exemplare beruht. Wenn wir aber diese bei der Rechnung gar nicht zu vermeidenden Fehler mit in Anschlag bringen, werden wir kaum daran zweifeln können, dass zur Zeit der Staufer die im Königreiche Sicilien übliche libra etwas grösser war als die, deren

1) Cherrier nimmt nach den Wägungen der beiden Pariser Exemplare 5,26 Gramm an : Huillard, Recherches 166 lässt die Unze 21,20, den Augustalis 5,30 Gr. wiegen und Blancard p. 218 gibt als mittleres Gewicht des letzteren obne nähere Begründung 5,22 Gr. an. Wenn er ferner als Durchschnitt des dem Augustalis nachgebildeten angiovinischen Regalis 5,27 Gr. (die Unze solcher Regalen also mit 21,08) ansetzen zu dürfen glaubt, so ist auch das bedenklich, weil sich die Berechnung auf zu wenige Exemplare der Regalen, nämlich nur auf drei, stützt.

2) Bei den Goldmünzen des deutschen Reichs ist die Grenze der Abnützung, bei welcher sie noch als vollwichtige zu behandeln sind, auf 5 Tausendstel des Normalgewichts festgesetzt. 
man sich noch jetzt dort gelegentlich bedient. Ich halte es in hohem Grade für wahrscheinlich, dass sie nichts anderes als noch das altrömische Pfund von 325,44 Gr. war, aus dem sich dann weiter nach unten als gesetzliches Gewicht ergeben würden

$$
\begin{aligned}
& \text { für die Unze } \frac{325,44}{12}=27,12 \mathrm{Gr} \text {. } \\
& \text { für den Tari } \frac{27,12}{30}=0,904,
\end{aligned}
$$

Ist dies freilich nur eine Hypothese, so wird sie doch dadurch unterstützt, dass, wie schon hier bemerkt werden mag, der Goldtari Friedrichs II. wirklich diesem für ihn ermittelten gesetzlichen Gewichte entspricht, d. h. dass er wirklich ${ }^{1}{ }_{30}$ Unze wog, insoweit Genauigkeit bei so kleinen Mengen mit den damaligen mangelhaften technischen Hilfsmitteln überhaupt zu erreichen war. Aber dasselbe gilt - immer unter der Voraussetzung, dass die normännisch-staufische libra nichts anderes als das römische Pfund war - auch ron dem Augustalis. Wir dürfen auch bei ihm wohl von Uebereinstimmung zwischen seinem gesetzlichen Gewichte von 6 Tari $(6 \times 0,904)=5,424 \mathrm{Gr}$. und seinem wirklichen mittleren Gewichte von 5,350 Gr. reden, indem letzteres nur um $0,074 \mathrm{Gr}$. hinter jenem zurückbleibt. Der Unterschied ist jedenfalls zu klein, als dass eine absichtliche Gewichtsminderung anzunehmen wäre, und die Mangelhaftigkeit der Wagen reicht vollständig zu seiner Erklärung aus.

In dieser Beziehung wenigstens erwuchs also den Unterthanen des Kaisers aus der erzwungenen Aufnahme der neuen Münze kein Schaden, da der Augustalis wirklich 6 Tari wog, und, wenn er für $1 / 4$ Unze Gold oder $7 \frac{1}{2}$ der ausgemünzten Tari angenommen werden musste, es sich noch fragen wird, ob nicht sein Goldgehalt diese anscheinende Verkürzung der Unterthanen an seinem Gewichte ausglich.

5. Der sicherste Weg zur Ermittlung des Goldgehalts der Augustalen wäre ja der, dass Jemand von den beati possidentes ein Exemplar der wissenschaftlichen Analyse opfere. Da dies aber schwerlich geschehen dürfte, bleibt nichts übrig als der Versuch, mit Hilfe der geschichtlichen Ueberlieferung zum Ziele zu gelangen, und diese ist glüeklicher Weise, was diesen Punkt betrifft, beredter als in Bezug auf die Einführung der neuen Münze.

In der oben (S. 402) erwähnten, ihrem Inhalte nach jedenfalls auch für die staufische Zeit giltigen Münzerordnung heisst es ${ }^{1}$ ):

1) Winkelmann, Acta imp. I, 766. Uebrigens scheint schon Garampi dieses Stück gekannt und benützt zu haben, wie ich aus den von Faraglia, Storia dei 
Augustales auri, qui laborantur in predictis siclis (non Brindisı und Messina), fiunt de caratis viginti et medio, ita quod quelibet libra auri in pondere tenet de puro et fino auro uncias $x$. tarenos vii $1 / 2$; reliqua vero uncia una et tareni viginti duo et medius sunt in quarta parte de ere et in tribus partibus de argento fino, sicut in tarenis.

Man verwendete also für die Augustalen 201/2karätiges Gold d. h. eine Legirung, die unter 24 Theilen aus 201/2 Theilen Feingold und $3 \frac{1}{2}$ Theilen anderer Metalle bestand 1 ), oder was dasselbe besagt, eine Mischung, in der auf das Pfund von 12 Unzen 10 Unzen $7 \frac{1}{2}$ Tari Feingold und 1 Unze 221/2 Tari zwölflöthigen Silbers (d. h. solchen Silbers, das zu einem Viertel mit Kupfer versetzt war) kamen.

Diese Legirung war besser als die bei den Goldtari gebrauchte (s. u.), aber die Thatsache, dass sie von der herkömmlichen abwich, wird neben dem Mindergewichte der Augustalen wesentlich dazu beigetragen haben, dass sich anscheinend der Verkehr nie recht mit dem neuen Zahlungsmittel befreundet hat und dass man fortfuhr, in der hergebrachten 'Weise nach Goldunzen und Goldtari zu rechnen. Die kaiserliche Regierung selbst verfubr nicht anders. Die Schuldverschreibungen, die Friedrich II. in den Jahren des Kampfes und der Finanznoth seit 1239 für auswärtige Bankiers machte ${ }^{2}$ ), sind sammt und sonders auf Goldunzen und Goldtari ausgestellt und die Steuern, die er im Königreiche ausschrieb, sind ebenfalls stets in Goldunzen angesetzt ${ }^{3}$ ). Und ebenso ging es unter seinen Nachkommen: eine ausserordentliche Steuer, die anfangs 1266 für Sicilien ansgeschrieben wurde, wurde in Goldunzen, Tari und Gran erhoben ${ }^{4}$ ).

Uebrigens lassen sich jene Angaben des dreizehnten Jahrhunderts über den Goldgehalt des Augustalis mit leichter Mühe in Grammen umsetzen. Jeder Augustalis, der mit dem normalen mittleren Gewichte von 5,350 Gr. die Münze verliess, enthielt zunächst

$$
\frac{5,350}{24} \times 201 / 2=4,57 \text { Gr. Feingold, }
$$

während der verbleibende Rest von $31 / 2$ Karat $=0,78$ Gr. zu drei Vierteln $=0,585$ Gr. aus Feinsilber und zu einem Viertel $=0,195 \mathrm{Gr}$.

prezzi (Napoli 1878) p. 25 nach Fusco, Intorno ad alcune monete Aragonesi (Nap. 1846) p. 18 citirten Stellen über das Metall der Augustalen und Tari schliesse.'

-1) E ist also zwar beinahe, aber doch nicht ganz genau, wenn Blancard p. 217. sagt, dass die Augustalen $\% / 7$. Feingold enthielten.

2) Winkelmann, De regni Siculi administratione p. 31 .

8) Vgl. Winkelmann, Acta imp. I, 630 (1238). 666 (1241 ?). 712 (1248?).

4) BWW. nr. 1427

Mittheilungen XV. 
aus Kupfer bestand 1). Da nun vier Augustalen eine uncia augustalium ausmachen sollten, erhielt man in ihr 18,28 Gr. Feingold.

6. Neben den Augustalen sind auch balbe Augustalen in Umlauf gebracht worden, wie die auf uns gekommenen Exemplare bezeugen. Bei Rycc. de S. Germ. da, wo er die Einführung jener erzählt, ist allerdings von ihnen ebenso wenig die Rede, als in der staufischen Münzerordnung; aber sie werden sowohl in staufischen als angiovinischen Mandaten gelegentlich erwähnt. Dafür jedoch, dass sie gleichzeitigen Ursprungs mit den ganzen Augustalen sind, spricht alle Wahrscheinlichkeit, vor Allem die völlig gleiche äussere Ausstattung, obwohl natürlich in kleinerem Massstabe (Durchmesser 1,60 Centimeter). Sie zeigen also auch auf der Seite, die ich für die Vorderseite ansehe, den Adler mit der durch seine Fänge getheilten Umschrift † FRIDE $\mid$ RICVS und auf der Rückseite die gleich gestaltete Büste des Kaisers mit der ebenfalls getrennten Umschrift IMPROM || CESARAVG.

Ihre Bedeutung für den Verkehr mag noch geringer gewesen sein als die der Augustalen und namentlich die Konkurrenz der von Alters her üblichen Goldtari wird sie nicht recht haben aufkommen lassen, um so weniger als ihr Werthverhältniss zu dieser allein wirklich verbreiteten Goldmünze (3 Tari 15 Gran) ein möglichst unbequemes war. Daher kommt es denn wohl auch, dass, wenn die Zahl der uns erhaltenen Augustalen nicht gerade eine sonderlich grosse zu nennen ist, die der halben noch viel kleiner ist, wenigstens der mir bisher zugänglich gewordenen. Denn ich kenne deren nur fünfzehn und zwar aus Berlin 2, Donaueschingen 1, Gotha 1, London 2, München 1, Neapel 4, Nürnberg 1, Palermo 2 und Paris 1. Diese schwanken in ihrem Gewichte, das unten den einzelnen Exemplaren beigesetzt ist, zwischen 2,60 und 2,70 Gr., also erheblich weniger als die ganzen Augustalen, und vertheilen sich auch nur auf zwei Stempel, bei deren Beschreibung ich mich kurz fassen kann, da die halben Augustalen, wie gesagt, nur verkleinerte Kopien der ganzen sind und für sie alles zutrifft, was von diesen im Allgemeinen zu sagen war.

I. V.: Adler, nach rechts vom Reschauer gekehrt. Umschrift ohne alle Punkte: + FRIDE $\mid$ RICVS

$R$. : Büste des Kaisers, rechts gekehrt. Umschrift: -IMPROM· CESARAUG.

Berlin $1(2,66)$ Berlin $2(2,64)$, Donaueschingen $3(2,67)$, London $1(2,656)$, London $2(2,656)$, Neapel Nr. $1134(2,613)$, Nr. 1135 (2,613), Nr. 1136 $(2,633)$, Nr. $1137(2,638)$, Nürnberg $(2,63)$, Palermo $1(2,65)$, Paris Nr. 1001 (2,60). - Vgl. Nr. 6 der Tafel nach Palermo 1.

1) Zur Vergleichung mag angeführt werden, dass ein deutsches Zwanzig. markstück 7,168 Gr. Feingold auf 7,695 Gr. enthält. 
II. V.: sonst wie I, aber rechts und links rom Kopfe des Adlers sind Punkte angebracht und RICVS ist sehr gesperrt.

$R$.: sonst wie I; doch ist die-Sehrift kleiner, SAR auseinander gezogen und $G$ so eingeengt, dass kein Raum für einen Schlusspunkt bleibt.

Gotha $(2,60)$, Mubehen (2,70), Palermo 2 (2,60). - Nach letzterem Nr. 7 der Tafel.

Als jetziges Durchschnittsgewicht des halben Augustalis ergiebt sich aus diesen Stücken 2,654 Gr. und als sein ursprüngliches Gewicht wenn wir auch hier etwa ein Procent auf die Abnützung rechnen, 2,680 Gr., mithin sogar eine Kleinigkeit mehr, als die Münze sowohl als Hälfte des Augustalis eigentlich haben musste, nämlich $\frac{5,350}{2}=$ 2,675 Gr., als auch wegen ihrer Gleichstellung im Gewichte mit 3 Tari, die König Karl bezeugt. Denn wenn wir von dem aus dem Münzgewichte des ganzen Augustalis gefolgerten Gewichte des Tari mit 0,891 Gr. ausgehen, hätte der halbe Augustalis auch nur 2,673 Gr. zu wiegen gebraucht. Freilich stellt sich das Verhältniss etwas anders, wenn wir der Vergleichung den aus dem römischen Pfunde abgeleiteten Tari mit seinen 0,904 Gr. zu Grunde legen, indem darnach das normale Gewicht des halben $\Lambda$ ugustalis 2,712 Gr. hätte sein müssen, also etwas mehr, als er anscheinend bei seinem Ausgange aus der Mänzstätte durchschnittlich zu haben pflegte. Aber auch in diesem Falle ist der Unterschied (0,032 Gr.) viel zu klein, als dass an eine absichtliche, von fiscalischen Erwägungen eingegebene Gewichtsminderung zu denken wäre, und alles in allem, wir haben ein Recht zu der Behauptung, dass auch der halbe Augustalis vollwichtig die Münze verliess.

Wie die Ausstattung, so war auch der Goldgehalt des halben Augustalis dem des ganzen vollkommen gleich. Wir nüssen das schon deshalb annehmen, weil die Münzerordnung über denselben gar nichts zu bemerken hat und weil auch Karl $I$. in seinen Verordnungen in Betreff der ganzen und halben Regalen, die den ganzeu und halben Augustalen entsprechen sollten, zwischen ihnen rücksichtlich des zu verwendenden Goldes keinen Unterschied macht ${ }^{1}$ ). Beide sollten in Gehalt und Gewicht sein, wie die Augustalen und halben Augustalen zur Zeit des verstorbenen Kaisers ${ }^{2}$ ).

7. Hat der Kaiser nach 1231 noch öfters A usprägungen seiner neuen Goldmlinzen vorgenommen? Ich möchte diese Frage weder be-

1) Del Giudice, Cod. I, 197 von 1266 : videlicet quod quelibet libra regalium et mediorum regalium contineat de auro puro in pondere uncias auri 10 et tarenos 7 et medium.

2) So noch 1.273 ian, 21 Arch. stor. Ital. Ser. 3 T, XXII, 10. 
jahen noch verneinen. Die Ueberlieferung lässt uns in dieser Beziehung vollständig im Stich und die Thatsache, dass verschiedene Stempel für jene Münze gebraucht worden sind, verträgt sioh sowohl mit der Annahme, dass sie alle gleichzeitig für die Prägung des Jahres 1231 gebraucht wurden, als auch mit der entgegengesetzten, dass sie zu Prägungen verschiedener Jahre dienten. Wollte man aber diejenigen Augustalen, deren Stempel künstlerisch vollendeter sind, früheren Jahren, und die, welche in dieser Hinsicht zurückstehen, den, späteren Jahren des Kaisers zuweisen, etwa als ob nun in Folge der politischen Bedrängniss, wie das sonst wohl vorkommt, auf das Aussehen der Münzen weniger Gewicht gelegt worden wäre, so lässt sich mit gleichem Rechte auch das umgekehrte Verhältniss behaupten und mit der Erwägung begründen, dass Geschicklichkeit und Fertigkeit der Stempelschneider doch mit den Jahren gewachsen sein muss. Am ersten würde auf wiederholte amtliche Prägungen geschlossen werden können, wenn die mittleren Gewichte der einzelnen Gruppen sich in einer absteigenden Linie bewegten; denn dann liesse sich wohl aunehmen, dass die durchschnittlich gewichtigeren Stücke aus früheren und die leichteren aus späteren Prägungen hervorgegangen seien, das heisst, dass die Finanznoth zur Verringerung des Gewichts geführt habe. Aber die Unterschiede jener Durchschnittsgewichte sind nicht gross genug, um aus ihnen eine solehe Folgerung zu ziehen, und sie wird zu Gunsten der anderen Aunahme, dass die Gewichtsunterschiede nur auf mangelhafter Technik bei der Ausbringung der einzelnen Stiicke beruhen, vollends fallen gelassen werden müssen, wenn wir berïcksichtigẹn, dass der Nachfolger der Staufen im Königreiche gar nichts davon weiss, dass es schwèrere und leichtere Augustalen oder solche von besserem und schlechterem Galdgehalte gab. Er kennt nur Augustalen und halbe Augustalen von ein em gesetzlichen Gewichte und Gehalte.

Meines Wissens kann für die Annahme mehrfacher Ausprägungen nur eins angeführt werden, nämlich dass es in der Münzerordnung heisst: Augustales auri, qui laborantur in predictis siclis, fiunt u. s.w. Hier weist : das Praesens allerdings auf eine noch fortdauernde Anwendung der angegebenen Vorșchriften hin und diese selbst würden ohnedem keinen rechten Sinn haben.

Aber wenn auch der Kaiser : selbst auf die weitere Ausprägung yon Augustalen und Halbaugustalen verzichtet haben sollte, so ist damit noch nicht erwiesen, dass überhaupt keine mehr stattgefunden haben könnte. Denn ans der Münzerordnung erfahren wir die interessante Thatsache, dass auch Privaten die Möglichkeit eingeräumt war, in den Münzstätten von Brindisi und Messina Gold auf ihre: Rechnung 
prägen zu lassen und zwar unter der Bedingung, dass sie für die Unze ausser den auf $4^{1 / 2}$ Gran berechneten Herstellungskosten noch 15 $\frac{1}{2}$ Gran an den Fiscus entrichteten, offenbar als Ersatz des Gewinnes, den er bei eigener Prägung gemacht haben würde. Beide Abgaben zusammen betrugen also $20 \mathrm{Gran}$, oder 1 Tari $d$. h. ungefähr $3^{1 / 3}$ Procent 1$)$. In wie weit von dieser Erlaubniss Gebrauch gemacht wurde, wissen wir nicht; es dürfte jedoch nicht ganz selten geschehen sein, da der Kaiser in einem Erlasse vom 19. Juli 1238 bei der Bestellung von Aufsehern über die Münzstätten darauf gesehen haben will, dass es Leute seien, qui nullum partecipium cum mercatoribus habeant 2). Betrügerische Manipulationen konnten namentlich dann stattfinden, wenn die Privaten, wie es nach der Münzerordnung gestattet war, selbst das zur Prägung Nöthige lieferten, statt es zu bezahlen.

8. Eine äusserst merkwürdige Erscheinung ist es, dass die alte sicilische Goldmünze, der tarenus a uri (Tari) nicht nur bei der Einführung der neuen fridericianischen Goldmünzen im Umlaufe als gesetzliches Zahlungsmittel belassen wurde und sich erhielt, sondern sogar seinerseits, wie aus den viel häufigeren Erwähnungen der Goldtari zu schliessen ist, ihre Einbürgerung hinderte. Das Werthverhältniss des Goldtari zum Augustalen war äusserst unhequem $\left(1: 7 \frac{1}{2}\right)$ und das zum halben Augustalis $\left(1: 3 \frac{3}{4}\right)$ noch mehr; das für die Tari verwendete Gold war, wie wir sehen werden, schlechter als das der Augustalen, aber die Macht der Gewohnheit wirkte stärker als alle Vortheile, die die Neuerung mit sich brachte, so dass eben nichts anderes übrig blieb, als das Bedürfniss des Verkehrs durch fortgesetzte Ausprägungen der bis 1231 allein in Gebrauch gewesenen Goldmünze zu befriedigen, und das geschah nicht nur unter Friedrich, sondern auch noch onter Karl I.

Der Namen des tarenus stammt von dem im neunten Jahrhunderte durch die Mauren eingeführten dirhem oder trihm (Plur. trahi), der den Werth eines Drittels des byzantinischen Solidus hatte ${ }^{9}$ ), und

1) Winkelmann, Acta imp. I, 766: Consuevit curia recipere pro qualibet uncia tam tarenorum quam augustalium, que laboratur in predictis siclis, grana $15 \frac{1}{2}$. Verumtamen mercator, qui facit laborari aurum suum in siclis ipsis, preter grana $15 \frac{1}{2}$ debet solvere alia grana $4 \frac{1}{2}$ pro qualibet uncia, quam laborari facit in siclis, pro expensis, que fiunt in labore uncie cuiuslibet etc. Wenn, wie wir weiterhin sehen werden, vor Grana $15 \frac{1}{2}$ wahrscheinlich, tarenum unum et aus. gefallen sein sollte, würden die Privaten im Ganzen nicht 1 Tari, sondern 2 Tari d. h. c. $6 \%$ srocent zu zahlen gehabt haben. . 2) Das. I, 637.

8) Amari, Storia dei Musulmani II,458. Vgl Faraglia Storia dei prezzi in Napoli p. 23 mit Berufung auf das mir nicht zugängliche Buch von Schiavo, ll tari d'oro. 
die normannischen Herrscher Siciliens übernahmen die Münze von ihren Vorgängern. Nun ist meines Wissens über den Goldgehalt dieser Tari bisher nichts bekannt geworden, das Gewicht jedoch war schon damals $1 / 30$ Unze 1). Indessen das thatsächliche Gewicht der von den Normannen in Sicilien, aber auch in Salerno geschlagenen Goldtari stellt in der Tabelle bei Engel, Nunismatique et sigillographie des Normands de Sicile p. 62 eine so ununterbrochene Abstufung von 2.42 bis $0,28 \mathrm{Gr}$. dar, dass die Scheidung derselben in viertel, halbe, einfache und mehrfache Tari eine Unmöglichkeit sein dürfte und die Annahme unabweislich ist, diese Münzen seien für gewöhnlich nicht gezählt, sondern zugewogen worden 2 ). Indessen die in Salerno geschlagenen Tari schwanken allein zwischen 0,83 und $0,90 \mathrm{Gr}$., so dass dies als das ungeführe Normalgewicht des Goldtari für jene Zeit anzusehen sein wird

Wie stand es nun mit Gewicht und Gehalt der unter Friedrich II. geprägten Goldtari? Die Beantwortung dieser Frage wird wiederum durch den Umstand erleichtert, dass Karl I. beides, ja sogar das Gepräge des fridericianischen Tarenus beibehielt und sich darüber wiederholt in seinen Verordnungen geäussert hat ${ }^{3}$ ).

9. Von tareni Friedrichs II. d. h. solchen, die ihm unzweifelhaft angehören oder auf seinen Namen gehen, sind mir allerdings nur wenige bekannt geworden. Doch wird daraus nicht auf ihre Seltenheit geschlossen werden können, denn ich muss bekennen, dass ich mich nach ihnen erst umsah, als sich meine ursprünglich auf die Augustalen beschränkte Untersuchung weiter ausdehnte. Immerhin ist es auffällig, dass jedes jener Stücke einen besonderen Stempel vertritt, wenn auch die Darstellung auf der Rückseite ihnen im Allgemeinen gemeinsam ist, nämlich ein Kreuz aus einem langen Fussbalken und einem gegen das obere Ende desselben angebrachten kurzen Querbalken mit der rechts und links davon vertheilten Beischrift:

1) Faraglia a, a. O. Wenn er aber p. 24 sagt, sie seien, d'oro sottilissimo* gewesen, so ist das von Vorne herein wenig wahrscheinlich.

2) Engel p. 64 betrachtet das von Wilhelm II. mit Knechtschaft und Gütereinziehung bedrohte, radere der Münzen als Hauptgrund für das Schwanken im Gewichte der normännischen Tari, muss aber zugeben, dass er nur selten Spuren davon an den Münzen selbst habe bemerken können.

3) 1271 Jar. 24 Minieri, Il regno p. 8 in einem ungenauen und leicht irreführenden Auszuge; 1271 Mai 7 das, 18; 1273 Jan. 21 Arch. stor. Ital. Ser. 3 T. XXII, 10: Tareni ..... (sint) in ea tenuta et modo, in quibus consueverunt feri temporibus retroactis (vorher imperatoris) in forma et cuneo consueto. De auro vero, quod in sicla laborabitur, quelibet libra de puro auro contineat uncias 8 et tarenos 5 , sicut consuevit hactenus contineri. 


\section{$\overline{\mathrm{IC}} \mid \overline{\mathrm{XC}}$ \\ $\mathrm{N} \mathrm{I}$ K A}

Die Hauptunterschiede dieser Seite liegen in der Gestaltung des Kreuzes, der Grösse der Buchstaben und der Form des Abkürzungszeichens, ob - oder —n_. Die Darstellung der Vorderseite ist mannichfaltiger. Auch das ist bemerkenswerth, dass die Mehrzahl jener Stücke nicht nur stark durch den Umlauf gelitten hat, sondern auch schon von Vorneherein verprägt ist $d$. $h$. der Stempel ist nicht genau in der Mitte des zur Prägung bestinmten Goldklümpchens aufgesetzt worden, das Münzbild daher nur unvollständig zum Ausdrucke gelangt. Die grosse Verschiedenheit des Gewichts endlich macht es unzweifelhaft, dass die vorliegenden Stücke nicht die gleiche Wertheinheit darstellen können, dass sie vielmehr theils einfache, theils mehrfache Tari sein sollen. Ich führe sie deshalb hier nach der Abstufung ihres Gewichts auf:

I. V.: In der Mitte ganz klein $\overline{\mathrm{FR}}$. Doppelte Umschrift zwischen drei Kreisen; von der inneren glaube ich SCL zu erkennen; die äussere ist verprägt und unleserlich.

R.: Kreuz mit der üblichen Beischrift. Abkürzungszeichen -. Umschrift theilweise erhalten, aber unleserlich.

Donaueschingen 4 (Durchmesser 1,35 C. - Gewicht 3,74 Gr.)

II. V.: In der Mitte $\overline{\mathrm{FR}}$ gross. Umschrift zwischen zwei Kreisen verprägt; zu erkennen $\mathrm{AVG} \ldots \mathrm{M}(\mathrm{P})$.

R.: Kreuz mit grosser Beischrift. Abkürzung durch -. Keine Umschrift.

Donaueschingen 5 (Dm. 1,20 C. - Gew. 3.28 Gr.) - Vgl. Nr. 12 der Tafel. Da das Stück trotz seines Gewichts klein ist, wird es verhältnissmässig dick sein.

III. V.: Adler, winzig klein. Umschrift unleserlich.

$\boldsymbol{R}$. : Kreuz mit Beischrift. Abkürzung durch -. Keine Umschrift. Gotha (Dm. I C. - Gew. 1,86 Gr.) - schlecht erhalten.

IV. V.: Adler, gekrönt, links (vom Beschauer) gekehrt, ganz roh. Umschrift verprägt: ... ICVS ...

R.: Kreuz mit Beischrift. Abkürzung durch -^-. Keine Umschrift. Donaueschingen 6 (Dm. 1 C. - Gew. 1,66 Gr.)

V. V.: Adler, anders als vorher, links gekehrt, gekrönt, ganz roh. Ein Punkt über jedem Flügel. Umschrift verprägt:.... ERIC.

$\boldsymbol{R}:$ Kreuz mit Beischrift. Abkürzung durch —^-. Umschrift verprägt.

Donaueschingen 7 (Dm. 1 C. - Gew. 1,43 Gr.).

VI. V.: Adler innerhalb eines Kreises, rechts (rom Beschauer) gekehrt, ungekrönt. Zu den Seiten des Halses 'S. (?) und 0. 
R.: Kreuz mit Beischrift. Abkürzungszeichen -.

Wien $2(\mathrm{Dm} .1$ C. - Gew. 1,37 Gr.) - vergoldetes Kupfer, also Fä lschung, aber doch wohl nach ächtem Vorbilde. - Vgl. Nr. 11 der Tafel.

VII. V.: Adler innerhalb eines Kreises, links gekehrt. Ueber dem Kopfe ein Punkt. Zu den Seiten des Halses 0 und V.

R.: Kreuz, an dessen Fussende rechts und links ein dicker Punkt mit Beischrift. Der oberste Theil der Darstellung ist abgeschliffen.

Wien 1 (Dm. 0,95 C. - Gew. 1,35 Gr.). - Vgl. Nr. 10 der Tafel.

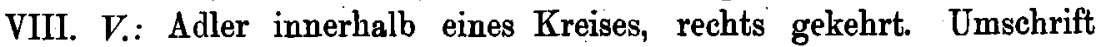
verprägt: .... R $\mathrm{RO}$.

R.: Kreuz, an dessen Fussende rechts und links ein Punkt, mit Beischrift, ohne Einschliessungsring. Abkürzung durch Wien 3 (Dm. 1 C. - Gew่, 1,32 Gr.).

IX. V.: Adler, ganz klein und äusserst roh. Doppelte Umschrift zwischen Kreisen, verprägt und versehliffen.

R.: Kreuz mit Beischrift innerh. eines Kreises. Abkürzung durch -. Donaueschingen 8 (Dm. 1 C. - Gew. 1,32 Gr.).

X. V.: In der Mitte $\because$ Umlaufend zwischen zwei Kreisen undeutliche Zeichen. Verprägt.

R.: Kreuz mit Beischrift innerhalb eines Kreises. Ablürzung durch -.

Donaueschingen 9 (Dm. 1 C. - Gew. 1, 25 Gr.). - Vgl. Nr. 9 der Tafel.

XI. V.: Adler, nach rechts gekehrt, gekrönt, gauz roh. Ueber jedem Flügel ein Punkt. Unschrift verprägt: .... ICVS.

R.: Kreuz mit Beischrift, innerhalb eines Kreises. In der Mitte des Schafts noch ein Knauf. Abkürzung durch -n-.

Donaueschingen 10 (Dm. 1 C. - Gew. 0,93 Gr.). - Vgl. Nr. 8 der Tafel.

Wenn einst die Taristücke Friedrich II. mehr beachtet werden sollten, die bisher wegen ihrer groben Arbeit und über die Augustalen einiger Massen übersehen worden sind, werden sich ohne Zweifel noch viel mehr für sie verwendete Stempel herausstellen und bei den grossen Abweichungen, die schon die hier aufgeführten vorweisen, wird angenommen werden können, dass der Zahl der Stempel auch die Zahl der Prägungen entspricht. Aber davon sind wir weit entfernt, ja es wird wohl überhaupt nie gelingen, die einzelnen Prägungen zeitlich bestimmen zu können. Nachweisbar ist nur eine, die vom September 1221, die zu Amalfi stattfand ${ }^{1}$ ). Die Stücke nun, auf denen ROM

1) Rycc de S. Germ. p. 342 : tareni novi cuduntur Amalfitani. Im folgenden Jahre wurden sie zu Gunsten von in Brindisi geschlagenen (Silber-)Denaren eingezogen, mit denen dann mehrfach gewechselt wurde. 
oder AVG erkennbar ist, sind dadurch schon der Zeit nach 1220 zugewiesen. Jedoch rücksichtlich der übrigen mag höchstens vermuthet werden, dass der Stempel I mit FR auf der Vorderseite, bei dem nichts bestimmt auf das Kaiserthum weist, den früheren Jahren Friedrichs, vielleicht sogar noch der Zeit vor seiner Berufung nach Deutschland (1212) angehört, die mit dem Adler aber, und das sind die meisten, wahrscheinlich aus den Jahren nach seiner Kaiserkrönung, vielleicht sogar erst nach 1231 stammen, das heisst aus denselban Jahrzehnten, in welchen der Adler auch auf den Augustalen Platz fand. In welche Periode der Stempel X mit der Rosette zu setzen sein möchte, bleibt völlig ungewiss; ich vernuthe aber, in Friedrichs Jugendjahre, da die Rosette auch schon auf Münzen seiner normännischen Vorgänger vorkommt 1).

Schliesslich ist nicht einmal sicher, ob alle Taristïcke, die Friedrich beigelegt werden, und selbst die, deren Gepräge auf ihn hinweist, wirklich von ihm herrühren. Denn Karl von Anjou ging in seinem Anschlusse an das fridericianische System soweit, dass er für die Tari nicht nur, wie schon bemerkt ist, bei dem Gewichte und dem Gehalte, das die Tari innerhalb desselben hatten, blieb, sondern sich auch nicht bedachte, seinen Tari dasselbe Gepräge zu geben, und das sogar noch im Jahre $1273^{2}$ ).

10. Welche von den oben angeführten Münzen wird nun der einfache tarenus auri Friedrichs II. sein? Dass auch unter Friedrich wie unter den Normannen, unter denen es sogar so kleine Goldstüke wie $1 / 4$ oder $1 / 3$ Tari gegeben zu haben scheint, Theile des Tari oder, was dasselbe heissen würde, gewisse Summen von Granen zur Ausprägung gelangten, lässt sich nicht erweisen ${ }^{3}$ ). Jene Münzen können also nur entweder einfache Tari oder mehrfache sein. Aber wo ist die Grenze zwischen ihnen? Die Entscheidung scheint deshalb sehr misslich, weil die meisten Stücke schlecht erhalten, stark abgenützt oder auch heschnitten sind und weil andererseits aus der Rohheit der Arbeit, die sehr von der der Augustalen absticht, zu schliessen ist, dass die Münzer auch auf die Genauigkeit des Gewichts keine allzu grosse Sorgfalt verschwendet haben werden.

Letzteres aber ist thatsächlich wenigstens bei dem einfachen Taro nicht der Fall gewesen. Denn ob wir davon ausgehen, dass der Au-

1) Engel, Numismatique Tab. VI nr. 16.19.

2) in forma et cuneo consueto, s. o. S. 422 Anm. 3. Abbildungen von Tari Karls auf Taf. III der Ann. de la Soc. franç. de numism. T. XV.

s) Wenn einmal 1241 (Winkelmann, Acta I, 534 Z. 9) von tareni auri 200 minus quarto die Rede ist, so ist damit nicht gesagt, dass das Viertel ausgeprägt war; es kann auch nur ein bequemerer Ausdruck für 5 Gran sein. 
gustalis 6 Tari wiegen sollte, und aus seinem Gewichte als Gewichttari $\frac{5,350}{6}=0,891$ Gr. folgern, oder ob wir die Unze als ein Zwölftel des altrömisch-sicilischen Pfunds mit ihren 27,12 Gr. zum Ausgangspunkte nehmen und darnach den Gewichtstari als ihren dreissigsten Theil auf $0,904 \mathrm{Gr}$. bestimmen, so oder so ist wohl kaum ein Zweifel daran möglich, dass das fridericianische Goldstück von $0,93 \mathrm{Gr}$. (Stempel XI) ein zufällig gut gemessener tarenus auri sein sollte. Wird diese Entscheidung durch jene salernitaner Stücke der normännischen Zeit im Gewichte von $0,83-0,90 \mathrm{Gr}$. unterstützt, so darf ich andrerseits für sie auch auf die Zustimmung Blancards hoffen, der in seiner schätzenswerthen Abhandlung S. 224 eine kleine Goldmünze Karl I. von 0,86 Gr. im Marseiller Cabinete ebenfalls für den eigentlichen Tari hält 1). Karl aber hat sich, wie erwähnt, auch im Gewichte der Tari ganz der Praxis Friedrichs II. angeschlossen, so dass die Deutungen jenes Goldstückes des Kaisers und dieses von Karl sich gegenseitig stützen. Mit anderen Worten: der fridericianische Goldtari war vollwichtig; er war im Gegensatze zu den Augustalen, die nur 6 Tari wogen, aber für $7 \frac{1}{2}$ ausgegeben wurden, auf das volle Gewicht ausgebracht, welches ihm nach seinem Namen und nach der Stellung des Tari im herrschenden Gewichtssysteme zukam; er wog wirklich einen Tari.

Man könnte einwenden, dass in diesem Falle ja der Kaiser von der Prägung der Tari nicht nur keinen Vortheil, sondern unmittelbaren Schaden gehabt haben müsste, insofern die Unkosten der Prägung ungedeckt blieben. In der That ist das aber, wie sich in anderem Zusammenhange zeigen wird, nicht der Fall gewesen und es wurde nicht nur jener Aufwand gedeckt, sondern obendrein ein nicht ganz unbeträchtlicher Gewinn erzielt und zwar, was vorgreifend gleich hier bemerkt sein mag, vermöge des für die Tari verwendeten Goldes das freilich nicht Feingold sein konnte, aber noch stärker legirt was als das der Augustalen.

Während nach Obigem über den einfachen Tari wohl kaum nocb Streit entstehen wird, ist rücksichtlich der anderen ganz in der Weise der Tareni gestalteten, aber schwerererı Goldmünzen Friedrichs II nicht so leicht eine Entscheidung darüber zu treffen, was sie darsteller sollen. Blancard hat, entsprechend seiner, wie ich glaube, begründeter Auffassung von jenem kleinen Goldstücke Karls, unter zwei anderen desselben, ebenfalls in Marseille, das eine mit 1,70 Gr. als Doppeltari

1) Huillard-Brèholles, Recherches p. 166 nimmt für den-Tari nur ein Ge wicht von $0,706 \mathrm{Gr}$. an. 
das andere mit 4,10 Gr., das jedoch stark abgenützt ist und deshalb von ihm in seinem ursprünglichen Bestande auf 4,30 geschätzt wird, als 5 Tari bezeichnet. Das leuchtet ein. Aber bei den jedenfalls ein Mehrfaches des Tari darstellenden Münzen Friedrichs ist, ebenso wie bei den der Normannen, die Abstufung des Gewichts eine so unregelmässige, dass man an einer reinlichen Scheidung derselben wohl verzweifeln möchte. Unter Berücksichtigung sowohl der unzureichenden Werkzeuge und einer gewissen Liederlichkeit der Müuzmeister als auch der Abnutzung und der Beschneidung kann man vielleicht das Stück mit 3,74 Gr. auch noch als 5 Tari gelten lassen, aber kaum mehr das von 3,28 Gr.: höchstens könnten es 4 Tari sein. Wenn ferner das von 1,66 Gr. allenfalls als Doppel hingehen mag, ist solche Werthung bei denen von 1,52-1,43 Gr. schon bedenklicher und bei denen mit 1,35-1,25 wohl geradezu unmöglich. Oder sollten etwa auch $1 \frac{1}{2}$ Taristücke geschlagen worden sein? Die einfachste Erklärung aller dieser Unregelmässigkeiten wird doch die schon oben angedeutete sein, dass man auf Genauigkeit des Gewichts bei den Mehrfachen des Tari auch zur Zeit Friedrichs keinen sonderlichen Werth legte, weil sie, wie zur Normannenzeit, gewogen worden sein werden: nicht darauf kam es bei den einelnen Stücken so sehr an, ob sie wirklich 2, 3 oder 5 Tari waren, als vielmehr darauf, einen wievielsten Theil sie nach ihrem Gewichte von der uncia tarenorum auri ausmachten. Die einfachen Tari dagegen mochten trotzdem auf Treu und Glauben von Hand zu Hand gehen, eben weil sie verhältnissmässig genau adjustirt waren. War das bei den Mehrfachen des Tari nicht der Fall, so wird darum doch nicht anzunehmen sein, dass bei ihnen eine absichtliche Verringerung des Gewichts stattgefunden habe, weil dies allem widersprechen würde, was sich aus unserer Betrachtung der Goldprägung Friedrichs überhaupt ergibt. Man mag einräumen, dass gerade hier bei den Tari zu einer solchen Verringerung, wenn einmal aus dem fiscalischen Münzrechte Kapital gemacht werden sollte, eine starke Verlockung gegeben war, eine um so stärkere, je grösser wahrscheinlich die Masse gerade der Goldtari war, die entweder die Regierung selbst schlagen musste, oder Private, wie es ihnen gestattet war, für ihre besonderen Bedürfnisse schlagen liessen. Aber eben das lässt sich nicht erweisen, dass eine derartige Ausnützung der Goldprägung im Allgemeinen und der Tari im Besonderen beabsichtigt war oder versucht wurde. Dass sie ihre Kosten einbringen musste, war selbstverständlich, und dass sie auch noch aut einen gewissen Gewinn berechnet war, ist begreiflich; aber es wird sich zeigen, dass dieser in so zu sagen durchaus 
legaler Weise erzielt wurde, überdies wahrscheinlich der herkömmliche war.

Was endlich das unter Friedrich, vielleicht auch schon unter den Normannen, für die Tari verwendete Gold betrifft, so ist schon bemerkt worden, dass es schlechter war als das der Augustalen. Denn ès war, wie wiederum die Münzerordnung berichtet ${ }^{1}$, nicht, wie bei diesen, 20 $\%_{2}$ karätig, sondern es hatte nur $16 \frac{1}{3}$ Karat, das heisst, ein Pfund $=12$ Unzen des zu den Tari verwendeten Münzgoldes enthielt nur 8 Unzen 5 Tari Feingold, dagegen 3 Unzen 25 Tari zwölflöthigen Silbers oder solchen Silbers, das wie bei den Augustalen zu drei Vierteln aus Feinsilber und zu einem Viertel aus Kupfer gemischt war. Karl I. gab dann den von ihm geschlagenen Tari denselben Goldgehalt ${ }^{2}$ ).

Daraus lässt sich denn auch der Gehalt des einzelnen Tari ermitteln. Unter der Voraussetzung, dass sein Durchschnittsgewicht ( $1 / 30$ Unze) für die Zeit Friedrichs - und für die Karls gilt dasselbe 0,90 Gr. war, enthält er an Feingold $\frac{0,90}{9 t} \times 16 \frac{1}{3}=0,6125$ Gr., während der Rest von 0,29 Gr. sich zu 3 Vierteln aus Feinsilber = $0,22 \mathrm{Gr}$. und zu einem Viertel aus Kupfer $=0,07 \mathrm{Gr}$. zusammensetzt. Der Goldgehalt des einzelnen Tari aber gibt uns ohne Weiteres auch den der so überaus häufig erwähnten, nur gewogenen, nie gemünzten uncia tarenorum auri als $30 \times 0,6125=18,37 \mathrm{Gr}$. - ein Betrag, der dem der uncia augustalium mit $18,28 \mathrm{Gr}$. Feingold so nahe kommt, dass man schwerlich irre gehen wird, wenn man annimmt, dass zwischen ihnen gerade in Bezug auf den Gehalt an Feingold völlige Gleichheit beabsichtigt war. Anders konnte es ja auch nicht sein, da der Augustalis den Werth von $7 \frac{1}{2}$ Tari, die aus 4 Augustalen bestehende uncia augustalium also denselben Werth wie 30 Tari oder eine uncia tarenorum haben sollte. Und wir dürfen wohl sagen, jene Gleichheit ward nicht blos beabsichtigt, sondern auch in Wirklichkeit erreicht, insofern der in der Berechnung hervortretende kleine Unterschied sich zur Genüge aus der Mangelhaftigkeit der Berechnung selbst erklärt und namentlich daraus, dass für die Gewichtsbestimmung des Tari zu wenig Material vorlag, diese selbst also nur als annähernd genau

j) Acta imperii I,766: Aurum tarenorum, quod laboratur tam in sicla Brundusii quam in sicla Messane, est caratis 16 et tercia, ita quod quelibet libra auri unciarum 12 tenet de puro et fino auro uncias 8 tarenos 5; relique vero uncie auri 3 et tareni 25 sunt in quarta parte de ere et in tribus partibus de argento noro.

2) S. o. S. 422 Anm. 3. 
gelten kann, wenn auch vorläufig schwerlich eine genauere zu erzielen sein möchte.

Augustalen, Halbangustalen und Goldtari von dem in den obigen Ausführungen festgestellten Gewichte und Gehalte waren also im Königreiche Sicilien neben einander während der letzten zwanzig. Jahre Friedrichs II. und unter seinen Nachfolgern im Königreiche Konrad I. (IV, 1251-1254), Konrad II. (Konradin, 1254-1258) und Manfred $\left.(1258-1266)^{1}\right)$ die gesetzlichen Zahlungsmittel in Gold, während allerdings sowohl die Regierung als auch der Privatmann bei grösseren Beträgen nach wie vor mit. Vorliebe nach der ungemünzten Goldunze rechnete, kleinere aber für gewöhnlich nicht in Augustalen, sondern in Goldtari und noch kleinere in den ebenfalls nicht gemünzten Gran auszudrücken pflegte. Das war also auch das System der Goldrechnung; das Karl von Anjou bei der Eroberung des Königreichs im Jahre 1266 vorfand und das er sich, wie schon öfters zu bemerken Gelegenheit war, zusammen mit der ganzen Verwaltungsordnung Friedrichs vorläufig so vollständig aneignete, dass er nur die Augustalen in Regalen umtanfte.

11. Die Einführung der Regales und halben Regales geschah durch die schon öfters angezogene Verordnung Karls vom 5. November $1266^{2}$ ), in der, nachdem Gewicht, Gehalt und Umlaufswerth derselben im Einzelnen festgesetzt ist und zwar so, dass sich in diesen Beziehungen vollständige. Gleichheit mit den zur Einziehung bestimmten Augustalen und halben Augustalen ergibt, am Schlusse zusammenfassend nochmals betont wird, prout angustales et medii angustales olim erant dicte tenute et ponderis et expendebantur hactenus per quantitatem predictam ${ }^{3}$ ). Insofern wäre also über diese angiovinische Neuerung nichts weiter zu bemerken.

Aeusserlich aber sind die Regalen von ihrem Vorbilde sehr verschieden, weil dessen imperialistische Gestaltung von einem Könige selbst-

1) Auf den Namen der beiden ersten scheint, wenn wir uns an die reiche Sammlụng des Museo nazionale zu Neapel (o. Catalogo III, 1, 11) halten, überhaupt kein Gold geschlagen zu sein. Von Manfred ist nưr eine Goldmünze da, nach ihrer Beschreibung zu urtheilen, ein Tari oder ein Mehrfaches desselben.

2) Del Giudice, Cod. I, 197. Vgl. über die Neuerung ausser Blancard auch Sambon in Ann. de la Soc. fianç. de numism. XV, $221 \mathrm{ff}$. mit Tab, III und Faraglia Storia, dei prezzi p. 26, der aber den Goldgehalt mit dem Gewichte der Regalen verwechselt zu haben scheint. Es hatte übrigens auch schon unter den Normannen Goldmünzen gegeben, die Regales hiessen, s. Huiḷ. Bréh., Hist. dipl. 1I, 520.

8) Aehnlich in der Verordnung von 1273, Arch. stor. Ital. Ser. 3 T. XXI, 10 : in tenuta et pondere, in quibus facti fuerunt augustales et medii augustales tempore quondam imperatoris. 
verständlich ebenso wenig beibehalten werden konnte wie der Name ${ }^{1}$ ). Ihre Vorderseite (ich folge einem mir aus Gotha mitgetheilten Exemplare von $5,3 \mathrm{Gr}$. Gewicht) ${ }^{2}$ ) zeigt die rechts gerichtete Büste des Herrschers mit dem von einer Agraffe auf der Schulter zusammengehaltenen Königsmantel und mit einer mittelalterlichen Krone, unter der eine Art Haube bis in den Nacken reicht; dazu die durch die Büste getheilte von einem Perlenrande umgebene Umschrift

$$
\cdot+\text { KAROL } \| \text { DEI : GRA } \text {. }
$$

Die Rückseite trägt nieht den kaiserlichen Adler, sondern einen mit Iilien besteckten Schild, dessen drei Ecken in die in der Mitte des oberen Schildrandes beginnende Umschrift hineinragend sie in drei Stücke zerlegen:

$$
+\mathbf{R}\|\mathrm{EX}: \mathrm{SI}\| \mathrm{CILI} \| \mathrm{E} \text {. }
$$

Der sofortigen Ausprägung der Regalen scheinen Hindernisse in den Weg getreten zu sein, unter denen wohl die chronische Geldnoth des Königs, die durch den folgenden Angriff Konradins noch erhöht wurde, obenan stehen mochte. Noch im Februar 1269 wurde der Sold für Aufgebotene in Augustalen angesetzt ${ }^{3}$ ). Die wirkliche Prägung der Regalen begann erst $1271^{4}$ ), bis zu Ende des Jahres war sie vollendet.

Die Umgestaltung der sicilischen Goldwährung durch die gegen 1278 erfolgte Einführung von ganzen und halben Goldcarolinen ${ }^{5}$ ) zu erörtern, das liegt ausserhalb der Aufgabe, die ich mir gestellt hatte Nur das Eine mag noch bemerkt werden, dass die Augustalen keineswegs rasch durch die Regalen verdrängt wurden. Wir haben eine Verordnung Karls ron 1278, in der er die Ausfuhr rohen oder bearbeiteten Edelmetalls verbietet und nur die der Carolenses aurei et ar. gentei et medaliae ( $d$. h. halbe Carolinen) ipsorum et Augustales gestattet ${ }^{6}$ ), so dass von letzteren damals noch ziemlich viele im Umlauf gewesen sein müssen. In demselben Jahre wird amtlich eine Zahlung von 300 Goldunzen in Augustalen angewiesen 7); sogar noch 1283

1) Anders bei den Tari, s. o. S. 425.

2) Blancard p. 218 nimmt, wie oberwähnt, 5,27 Gr. als Durchschnitt an; er stützt sich unter Einrechnung der Abnützung auf drei Exemplare: in Wien von 5,15 - in Paris von 5,20 und in Marseille von 5,22 Gr. Das erste soll sehr schlecht, das letzte gut erhalten sein.

s) BFW. 14435.

4) Verordnung 1271 Mai 7. Minieri, Il regno p. 18.

6) Blancard in Revue numism. Nouv. Sêr. IX, 221. Schon 1271 waren Silbercarolinen (Carolenses argenti) eingeführt worden, von denen 60 den Werth einer Goldunze haben sollten, das Stück also gleich $1 / 2$ Goldtari. Das. p. 229.

в) Das. p. 227.

7) Syllabus monum. I, 170. 
zahlte die königliche Kammer unterschiedslos Carolenses und Augustalen aus $\left.{ }^{1}\right)$ und 1284 schärfte der König neuerdings ein, dass der Augustalis für $71 / 2$ Tari angenommen werden müsse ${ }^{2}$ ). Doch mag es wohl sein, dass, wie Blancard vermuthet, für die Regalen selbst noch gelegentlich die missbräuchliche Bezeichnung Augustalen weiter gebraucht wurde, und das dürfte nicht auffällig sein, da beide dem Wesen nach durchaus dasselbe bedeuteten.

12. Wenn ich nun den Versuch mache, auf dessen Erfolg ich bei dieser Untersuchung eigentlich das Hauptgewicht lege, nämlich den Werth der fridericianischen Goldmünzen und zwar sowohl ihren Metallwerth als ihren Dmlaufswerth zu bestimmen, so geschieht dies hauptsächlich zu dem Zwecke, um auf diesem Wege endlich einmal einen festen Anhalt zur Beurtheilung der wichtigsten Einzelheiten in Friedrichs Steuer- und Finanzwesen, der Besoldungsverhältnisse seiner Beamten und seiner viel bewunderten und viel geschmähten Verwaltung überhaupt, endlich auch des Handels und Verkehrs Unteritaliens für diese Zeit zu erlangen. Denn alle die zahlreichen Nachrichten, die wir über diese Dinge besitzen, schweben vollständig in der Luft, so lange ein fester Massstab zur Vergleichung des damaligen Münzwerths fehlt. Wie weit aber gehen die Sehätzungen jener Münzen und vor Allem auch der Unze, auf der alles beruht, bis jetat auseinander!

Huillard-Brèholles, Recherches sur les monuments, Append. II, giebt dem Goldtari einen inneren Werth von 2,55 Fr. und für seine Zeit einen solchen von 3,70 Fr., mithin der Unze als dem dreissigfachen den Werth von $761 / 2$ bez. 111 Fr. - Cherrier, Histoire de la lutte (2 éd.) II, 32 dagegen, der das aus den zwei Augustalen in Paris abgeleitete mittlere Gewicht derselben mit 5,26 Gr. zu Grunde legt, schätat die Unze als das Vierfache des Augustalis, den Gramm zu 3 Fr. berechnend, nur auf 63,12 Fr. und Andere kommen ihm ziemlich nahe. So Amari, La guerra II, 402 mit dem Ansatze von $61,50 \mathrm{Fr}$. und Blancard in seinem oft angezogenen Aufsatze S. 223 mit dem von $62,76 \mathrm{Fr}$. und es verlohnt sich zu sehen, wie gerade dieser Forscher dazu gelangt ist, da er allein von allen Genannten richtig erkannt hat, dass vor Allem auch der Goldgehalt berücksichtigt werden muss.

Indem nach seiner Berechnung die Unze Regalen - und für die Augustalen müsste nach Obigem ganz dasselbe gelten - 21,08 Gr. wog (s. o. S. 415 Anm.), setzt er die nach seiner nicht ganz genauen Annahme darin enthaltenen

1) Faraglia, Storia dei prezzi p. 27 n. 3.

2). Das. n, 1. 
18 Gr. Feingold . . . . . . . . . $=62,-$ Fr.

2,31 Gr. Silber . . . . . . . . . . $=0,51$,

0,77 Gr. Kupfer . . . . . . . . . = $=0,004$,

Zuschlag für den höheren Silberwerth . . = 0,25 ,

insgesammt also die Unze Regalen . . $=62,76$ Fr.

Indessen wir können Blancards Ergebniss nicht als überzeugend betrachten, weil das von ihm angenommene Durchschnittsgewicht des Regalis (Augustalis), die Grundlage der ganzen Rechnung, nicht mit dem von uns aus viel zahlreicheren Wägungen ermittelten und, wie wir deshalb bis auf Weiteres glauben müssen, richtigeren übereinstimmt, der Unterschied aber immerhin ziemlich beträehtlich ist. Dazu komnit noch, dass er auch der Augustalenunze ein Gewicht von 30 Tari gibt, was ein Irrthum ist. Denn der Augustalis sollte wohl den Werth von $1 / 4$ Unze haben, aber nur das Gewicht von 6 Tari oder $1 / 5$ Unze, und das ist auch von den Anderen, die sich in dieser Beziehung geäussert haben, übersehen worden.

13. Es ist von Vorneherein zu erwarten, obwohl es bisher nicht beachtet wurde, dass der Werth der Unze, die aber immer nur eine Rechnungsmünze war, möglicher Weise doch sehr verschieden gewesen sein kann, je nachdem man eine uucia auri (puri, fini) oder eine uncia augustalium oder eine uncia tarenorum meinte. Während unter der ersten Reingold verstanden wurde, bezogen sich die beiden anderen auf eine Legirung, und zwar war diese bei ihnen nicht eine gleich starke. Dieser Unterschied wird also überall, wo von der Unze als Geldwerth die Rede ist, wohl zu berück-ichtigen sein, wenn man nicht in Irrthümer verfallen will, die unter Umständen eine ziemliche Tragweite haben können.

Den heutigen Metallwerth der sicilischen uncia auri zu bestimmen, macht keine Schwierigkeit, nachdem das Gewicht einer Unze überhaupt auf 27,12 Gr. festgestellt werden konnte. Da nach dem Satze von 1392 Mark für das Ptund (500 Gr.) Feingold, zu welchem die deutsche Reichsbank solches zu kaufen rerpflichtet ist, ein Gramm heute den Preis von 2,78 M. hat, ist der heutige Metallwerth dieser Unze $75,19 \mathrm{M}$.

Die Werthe der beiden anderen Unzen zu berechnen, ist zwar etwas umständlicher, aber ebenfalls nicht schwierig, weil die dazu nöthigen Elemente, das Gewicht und der Gehalt der Tari und der Augustalen, schon oben gegeben werden konnten. Es muss aber hier gleich nochmals darauf hingewiesen werden, dass der Gehalt an Feingold bei der 30 Tari wiegenden uncia tarenorum sich dem Gehalte an Feingold bei der nur 24 Tari wiegenden uncia augustalium so 
nahe kommend erwiesen hat, dass wir wohl Grund haben, von Uebereinstimmung zwischen ihnen zu reden ${ }^{1}$ ).

14. Beginnen wir mit der Feststellung des Metallwerths des Tari, als der älteren Münze, und der uncia tarenorum.

Rechnen wir nun das Pfund Feingold wieder zu 1392 M. und das Pfund Feinsilber zu dem augenblicklichen (24. Nov. 1893), allerdings sehr unsicheren Marktpreise von $48 \mathrm{M}$. und gestatten wir uns den Werth des bischen Kupfers im Tari $(0,07$ Gr.) ganz ausser Betracht zu lassen, so würde sich für den Tari ergeben

$$
\begin{aligned}
& 0,6125 \mathrm{Gr} \text {. Gold im Werthe von } 1,70 \mathrm{M} \text {. } \\
& 0,22 \text { "Silber " } " 0,02 \mathrm{M} \text {. } \\
& \text { und als heutiger Metallwerth }=1,72 \mathrm{M.}^{2} \text { ) }
\end{aligned}
$$

Die aus 30 solcher Stücke gebildete uncia tarenorum die $30 \wedge 0,61=$ 18,37 Gr. Feingold enthielt, hätte darnach einen inneren Werth von 51,60 M. ${ }^{2}$ ) gehabt. Aber das kann nicht die Geltung gewesen sein, zu der die Regierung sie, und entsprechend den einzelnen Tari, in den Verkehr brachte, da sie sonst nicht auf ihre Kosten gekommen wäre, geschweige denn aus der Prägung irgend einen Gewinn gezogen hätte.

15. Ein günstiger Zufall verhilft uns auch zu den für die Berechnung des Verkehrswerths der Tari nöthigen Faktoren.

Wir besitzen eine äusserst interessante Zusammenstellung der Vortheile, die Friedrich 1I. aus jeder Silberprägung seiner letzten Jahrzehate gezogen hat ${ }^{3}$ ). Eine solche in Betreff seiner Goldprägungen fehlt allerdings meines Wissens, aber es liegt in der uns schon so oft förderlich gewordenen Ordnung für die Münzstätten von Brindisi und

1) S. oben S. 428 .

2) Huillard p. 166 schrieb dem Tari ein Gewicht von nur 0,706 Gr. zu, setzte aber seinen jnneren Werth auf 2,55 Fr. an - viel zu hoch, indem er wahrscheinlich nicht berücksichtigte, dass das Gold eben nicht Feingold ist. Blancard p. 223, der für die uncia tarenorum ein Gewicht von 25,85 Gr. annimmt, berechnet ihren Werth in folgender Weise:

$$
\begin{aligned}
& \text { 17,60 Gr. Feingold . . . 60,62 Fr. } \\
& \text { 6,20 Gr. Feinsilber . . . . 1,37 Fr. } \\
& \text { 2,05 Gr. Kupfer . . . . . 0,01 Fr. } \\
& \text { Höherer Silberwerth . . . 0,68 Fr. } \\
& \text { Zusammen 62,68 Fr. }
\end{aligned}
$$

Nach Blancard ist also die uncia tarenorum der uncia regalium gleichwertig, die er auf 62,76 Fr. 'berechnet hatte. Der angiovinisebe Tari aber, und für den staufischen gilt ganz dasselbe, würde nach ihm einen Werth von 2,09 Fr. darstellen - ein Ergebniss, mit dem sich das meine nahezu deckt.

8) Heransgegeben von Blancard in Revue numism. Nouv. Sér. IX, 305 und mit Besserung einiger Lesarten Winkelmann Acta I,763. 
Messina wenigstens die kurze Angabe vor ${ }^{1}$ ), dass der Regierung selbst eine uncia tarenorum auf 28 Tari $2 / 3$ Gran, eine uncia augustalium auf 27 Tari 18 Gran zu stehen kam (valet), worunter doch wohl eben nichts anderes als der Preis des Metalls selbst zu verstehen sein wird. Der ihr aus der Münzung einer uncia tarenorum - auf die Augustalen wird später zurückzukommen sein - erwachsende Vortheil betrug darnach 1 Tari $19 \frac{1}{3}$ Gran oder, di die Unze 600 Gran hatte, circa $6 \%$ Procent, oder, wenn wir dafür den zunächst allein bekannten Metallwerth des Taro einsetzen: 3,38 Mark. Es ist nun sehr auffällig, dass nach derselben Münzerordnung der Fiscus sich bei Ausprägungen sowohl von Augustalen als von Tari für Rechnung der Privaten (s. o. S. 421) den ihm entgehenden Gewinn angeblich nur mit 15/2 Gran vergüten liess, also bei den Tari mit weniger als der Hälfte dessen, was er aus eigener Münzung gezogen haben würde. Ich vermuthe deshalb, dass an der betreffenden Stelle: "tarenum unum grana 151/2 " zu lesen ist. Das würde nach dem Metallwerthe $3 \mathrm{M}$., nach dem Verkehrswerthe 3,20 M. ausmachen, so dass der mittelbare Verlust des Fiscus in diesem Falle unbedeutend gewesen sein würde.

Was die Unkosten betrifft, so weiss man wieder aus der Münzerordnung, dass sich der Fiscus, wenn er für Rechnung der Privaten münzte, dieselben mit $41 / 2$ Gran auf die Unze ersetzen liess (s. o. S. 421). Man kann annehmen, dass er bei Prägung für sich selbst schwerlich mehr aufgewendet haben wird. Jene $41 / 2$ Gran des für die Tari verwendeten Golds hatten einen Werth von 38,7 oder sagen wir der Einfachheit halber 39 Pfennigen.

Nun lässt sich auch angeben, welches der mindeste Preis war, zu dem die uncia tarenorum von der Regierung für den Verkehr berechnet worden sein wird. Denn es betrug

\begin{tabular}{lllr} 
ihr Metallwerth & $\cdot$ & $\cdot$ & $51,60 \mathrm{M}$ \\
der Gewinn an ihr & $\cdot$ & $\cdot$ & $3,38 \mathrm{M}$. \\
die Unkosten & $\cdot$ & $\cdot$ & $0,39 \mathrm{M}$. \\
\hline mithin ihr Verkehrswerth & $\cdot$ & $55,37 \mathrm{M}$.
\end{tabular}

und der des einzelnen Tari als des dreissigsten Theils dieser Unze darnach $\frac{0 \tilde{0}, 37}{30}=1,841 / 2 \mathrm{M}$, endlich der des nicht gemünzten Gran, nach dem aber häufig genug gerechnet wurde, wieder als des zwanzigsten Theils des Tari, ungefähr 9 Pfennige.

16. In ähnlicher Weise werden sich auch die Werthe der Augustalen, nämlich ihr Metallwerth uvd der ihnen amtlich beigelegte Werth, ermitteln lassen.

1) Winkelmann I, 767 Z, 39 . 
Lassen wir nun wieder als Preis für das Pfund Feingold 1392 M., für das Pfund Feinsilber $48 \mathrm{M}$. gelten, während die sehr kleine in Augustalis enthaltene Menge Kupfers auch hier wieder füglich vernachlässigt werden kann, so wird sich sein Metallwerth aus folgenden Posten zusammensetzen:

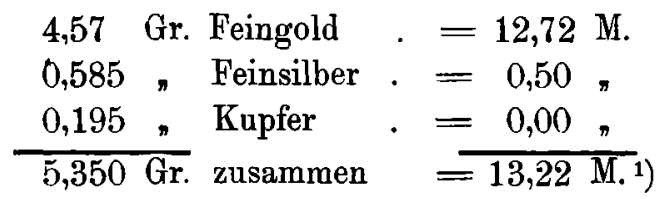

Da ein Augustalis 6 Tari wog, hätte ein Tari vom Golde desselben den Werth von 2,20 M., ein Gran den von 10 Pf. gehabt. Solche wurden jedoch nicht ausgemünzt und können deshalb hier ausser Betracht bleiben. Das aber stellt sich schon hier mit Bestimmtheit heraus, dass das Publicum dadurch, dass der Augustalis uur 6 Tari wog, während er 71/2 Goldtari (s. u.) gelten sollte, jedenfalls nicht geschädigt wurde. Denn der Werth seines Goldes kam dem der 71/2 Goldtari durchaus gleich, die auch $0,61 \times 71 / 2=4,57$ Feingold enthielten.

Der Augustalis wurde allerdings nicht zu jenem Werthe ausgegeben, konnte es auch nicht, sondern, wie es bei Rycc. de S. Germ. vielleicht im Anschlusse an den Wortlaut der kaiserlichen Einführungsverordnung von 1232 heisst, pro quarta uncie. Daraus ist zwar nicht ersichtlich, welche Art von Unze gemeint war, ob ein Viertel der Unze Feingold = 18,80 M., was ganz ungeheuerlich gewesen wäre, oder einer vollwichtigen Unze von dem für die Augustalen verwendeten Münzgolde - in welchem Falle der Augnstalis für 16,52 M. ausgegeben sein würde - oder endlich einer Unze der Tari. Letztere kann aber in der That allein gemeint sein, denn erstens gab es ja keine andere Goldmünze, die mit den Augustalen in Gleichung hätte gestellt werden können, und zweitens sagt Karl I. in seiner Verordnung von 1266 unter ausdrücklichem Hinweise auf das unter seinen staufischen Vorgängern Uebliche: quilibet (regalis) pro tarenis septem et medio expendatur. Nun konnte der Augustalis aber natürlich nicht zu dem Werthe umlaufen, den das Gold der 71/2 Tari hatte, sondern zu dem, den sie im Verkehre hatten oder zu dem sie von der Regierung ausgegeben wurden, und es ergibt sich mithin, da der Verkehrswerth des einzelnen Tari 1,84 $\frac{1}{2}$ M. betrug, dass desn Augustalis amtlich ein Umlaufswerth von $13,84 \mathrm{M}$. (bei 13,22 M. Metallwerth) beigelegt war. Die uncia augustalium, die durch 4 solcher Goldstücke gebildet wurde,

1) Es ist also viel zu hoch gegriffen, wenn Huillard, Recherches p. 166 den inneren Werth des Augustalis auf 19 Fr. schätzt. 
sollte demnach $55,36 \mathrm{M}$. gelten ${ }^{1}$ ), während $\mathrm{hbr}$ innerer Werth sich nur auf $4 \times 13,22=52,88 \mathrm{M}$. belief.

17. Zwei Umstände fordern bei diesem Ergebnisse zu besonderer Betrachtung auf. Der eine ist die vollständige Uebereinstimmung des der uncia augustalium und der uncia tarenorum gegebenen Verkehrswerths $(55,36$ M.) welche der schon vorher betonten Gleichheit ihres Gehaltes an Feingold $(18,28$ und 18,37 Gr.) trotz ihrer verschiedenen Legirung entspricht; der andere ist der nicht unbeträchtliche Unterschied zwischen Metallwerth und Verkehrswerth bei beiden.

Was jene Uebereinstimmung des Goldwerths betrifft, so konnte sie natürlich dem Verkehre nur förderlich sein. Aber bei der Verschiedenheit in der Güte des für beide Münzsorten verwendeten Golds hat sie nur auf künstliche, um nicht zu sagen, auf gewaltsame Weise erreicht werden können, nämlich eben dadurch, dass die uncia augustalium um ein Fünftel leichter angesetzt wurde als die uncia tarenorum. Sie wog eben nicht 30 wie diese, sondern nur 24 Tari und es war nur eine von dem amtlich dem Augustalis zuerkannten Werthe hergeholte Fiktion, wenn sie trotzdem als uncia bezeichnet wurde. Im anderen Falle, wenn man der Augustalenunze das Gewicht von 30 Tari gleich der gewöbnlichen Unze gegeben hätte oder, was dasselbe bedeutet, wenn nicht vier, sondern füuf Augustalen auf sie gerechnet worden wären, wäre die Ungeheuerlichkeit herausgekommen, dass ihr Metallwerth grösser gewesen wäre, als ihr Verkaufswerth, der seinerseits dadurch festgelegt war, dass der Augustalis eben ein Viertel der Tari-Unze gelten sollte. Eine Unze von 30 Tari des Augustalengolds $(5 \times 13,22)$ hätte den Werth von $66,10 \mathrm{M}$. gehabt, während eine Unze von vier gemünzten Augustalen im Verkehre nur 55,36 M. galt.

Die zweite Wahrnehmung ist vielleicht noch auffälliger. Während der Unterschied zwischen Metallwerth und Verkehrswerth sich bei der uncia tarenorum auf 3,77 M. belief, betrug er bei der uncia augustalium nur 2,48 M. Die kaiserliche Regierung begnügte sich bei ihr mit einem viel kleineren Gewinne. Das ist bei der auf anderen Verwaltungsgebieten deutlich genug hervortretenden Fiscalität derselben so überraschend, dass vielleicht nicht Jeder von der nächstliegenden Erklärung befriedigt sein wird, nämlich dass ohne solche Einschränkung eben die höchst wünschenswerthe Gleichheit im Verkehrswerthe der beiden Unzenarten nicht hätte erzielt werden können.

1) Von Allen, die sich mit der Schätzung des Augustalis befasst haben, hat Huillard a. a. 0 . sich also am Weitesten vergriffen, indem der Umlaufswerth der Augustalenunze nach ihm (s. o. S. 431) sich auf $111 \mathrm{Fr}$. gestellt hätte. 
18. Mit nicht geringer Freude ist es deshalb zu begrüssen, dass sich hier die Möglichkeit einer Prüfung der bisherigen Ergebnisse auf ihre Richtigkeit bietet. Noch erwünschter wird es freilich sein, wenn die Probe schliesslich stimmen sollte.

Diese Gegenrechnung wird durch die beiden Angaben der Münzerordnung ermöglicht, dass der Regierung das Metall jeder Unze der Augustalen auf 27 Tari 18 Gran zu stehen $\operatorname{kam}^{1}$ ), also ihr 2 Tari 2 Gran abwarf - das wäre etwas mehr als bei den Goldtari, nämlich 7 Procent -, und dass sie sich bei Privatprägungen $4 \frac{1}{2}$ Gran für ihre Unkosten auf die Unze ersetzen liess. Aber die auf diese Angaben sich stützende Rechnung scheint zunächst keineswegs zu einer Bestätigung unsers früheren Ergebnisses zu führen. Es betrügen nämlich darnach im Tarigelde:

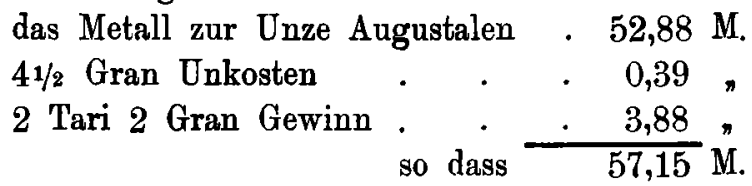

und nicht 55,36 M. der geringste Werth gewesen wäre, zu dem die Regierung die uncia augustalium in den Verkehr hätte bringen können.

Dieser Widerspruch lehrt, dass irgendwo ein Fehler in unseren Voraussetzungen steckt; es fragt sich nur, an welcher Stelle: ob in dem vorher aus dem Metallgehalte berechneten Umlaufswerthe der Augustalen oder in jenen Angaben der Münzerordnung in betreff von Gewinn und Unkosten bei ihrer Ausprägung, auf welchen diese zweite Berechnung beruht. Da nun ersteres durch die völlige Uebereinstimmung des Goldgehalts und Umlaufwerths der uncia augustalium mit dem der uncia tarenorum ausgeschlossen zu sein scheint, die schwerlich eine zufällige sein wird, sondern vielmehr sachlich geboten war, kann ich mir deshalb jene Abweichung der beiden Rechnungsergebnisse nur durch die Annahme erklären, dass sich in die Zahlenangaben der Münzerordnung selbst irgend ein Fehler eingeschlichen hat, sei es durch Schuld des Abschreibers, sei es schon durch ein Versehen desjenigen, von dem sie herrühren. Die Differenz würde z. B. gänzlich schwinden, wenn wir annehmen dürften, dass in der Angabe der Summe, auf welche der Regierung die uncia augustalium zu stehen kam, statt 27 (XXVII) 28 (XXVIII) Tari 18 Gran zu lesen wäre. Dann würde dem entprechend ihr Gewinn nicht 2 Tari 2 Gran, sondern nur 1 Tari 2 Gran betragen und wir gelangen zu folgender Aufstellung:

1) S. o. S. 434 . 


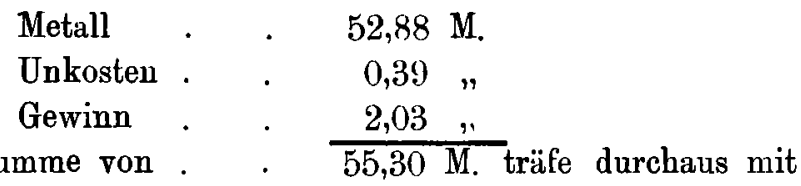
und die Gesammtsumme von . . 55,30 M. träfe durchaus mit den 55,36 M. zusammen, die sich uns auf anderem Wege als der von Amtswegen der uncia augustalium beigelegte Kurswerth ergeben hat. Ja noch mehr: nun deckt sich auch die durch die erste Methode ermittelte Differenz von 2,48 M. zwischen dem Metallwerth und dem Kurswerth der Unze Augustalen mit den 2,42 M., die nach der Korrektur der Münzerordnung als Summe von Gewinn und Unkosten bei einer solchen Unze in Ansatz zu bringen sind. Es scheint mir deshalb keinem Zweifel zu unterliegen, dass die Lesart der Münzerordnung in der That einer Besserung in jenem Sinne bedarf ${ }^{1}$ ), mit deren Vollzug dann alles in die beste Ordnung kommt.

19. Wie sehr die kaiserliche Regierung bestrebt war, die Verbreitung der Augustalen und ihre bereitwillige Aufnahme bei den Unterthanen zu befördern, sie diesen gewissermassen zu empfehlen, lässt sich schon aus der Thatsache erkennen, dass sie für sich bei dieser Münzsorte einen kleineren Gewinn beanspruchte als bei den Tari and dass sie der uncia augustalium, wie wir sahen, nur denselben gesetzlichen Werth im Verkehre beilegte wie der alten uncia tarenorum, obwohl ihr Metallwerth um 1,28 M. höher war als der der letzteren. Und nicht oft genug kann betont werden, dass wenn der einzelne Augustalis im Gewichte nur 6 Goldtari gleichkam, aber für $71 / 2$ genommen werden musste, die Unterthanen auch dadurch nicht zu Schaden kamen, indem sein Mindergewicht durch die bessere Beschaffenheit seines Goldes vollständig ausgeglichen wurde. Denn mit benerkenswerther Geschicklichkeit hat man es, wie gesagt, einzurichten gewusst, dass der Augustalis genau so viel Feingold enthielt als die 71/2 Tari, denen er gleich gewerthet war $(0,61 \times 71 / 2=4,57)$ und ebenso die uncia augustalium von 24 Tari Gewicht genau so viel als die uncia tarenorum, die doch 30 Tari wog ${ }^{2}$ ). Aber die Macht mehrhundertjähriger

1) Dass noch an einer anderen Stelle, rücksichtlich dessen, was sich die Regierung bei Privatprägungen für den ihr entgehenden Gewinn zahlen liess, an der Münzerordnung eine Korrektur wahrscheinlich nothwendig ist, ist S. 434 bemerkt worden. Aber selbst dann, wenn für die Unze Augustalen nicht 151/2 Gran, sondern 1 Tari $\left.15^{1}\right|_{2}$ Gran zu zahlen waren, wie wir das für die Unze Tari vermutheten, in unserm Gelde $=3,20 \mathrm{M}$., hätte die Regierung allerdings mehr erhalten, als ihr aus eigener Prägung erwachsen wäre.

2) Nach unserer Berechnung enthielt der Augustalis für 12,72 M. Feingold, und die $\left.7^{1}\right|_{2}$ Goldtari für 12,75, also gleich viel. Wegen der Unzen.s. o. S. 435. 
Eingewöhnung in ein Münzsystem ist eine gewaltige und eben deshalb wird Kaiser Friedrich II., als er neben die altgewohnte Münze eine neue zu seiner besonderen Verherrlichung bestimmte setzen wollte, trotz jenen Ausgleichungen im Jahre 1232 die strengen Strafandrohungen für nöthig erachtet haben, um ihre Annahme bei seinen Unterthanen zu erzwingen.

Der Versuch, die Augustalen im Königreiche sozusagen heimiseh zu machen, hätte gelingen können, wenn gleichzeitig die Goldtari aus dem Verkehre gezogen worden wären, musste aber nothwendig scheitern, wenn jene mit diesen in Konkurrenz gehracht wurden. Zwei gesetzliche Zahlungswittel neben einander, deren Einheiten im Werthverhältnisse von 1:71/2 standen, das war an sich ein Unding! Dazu kam dann noch die verwirrende Uebertülle der Münzsorten. Man versetze sich nur in eine Lage, in der, ganz abgesehen von den gewiss noch in grossen Massen vorhandenen Theilstücken des Tari aus der normännischen Zeit, der Tari selbst zu 1,84 M. und seine Mehrfachen zu etwa 3,68.7,36 und vielleicht auch zu 9,20 M. neben den halben Augustalen zu 6,92 M. und den ganzen zu 13,84 herliefen. In diesem Wettbewerb aber zog die neue Münze den Kürzern. Trotzdem dass sie manches für sich hatte, namentlich auch, dass sie nicht erst gewogen zu werden brauchte, und obwohl die staatliche Autorität ihr mit voller Wucht zu Hilfe kam, hat sie sich doch nie recht einzubürgern vermocht und sie ist schon ein Vierteljahrhundert nach dem Tode Friedrichs, nachdem auch noch Karl von Anjou mit ihr einen Versuch gemacht hatte, wieder aus dem Verkehre verschwunden, ohne eine andere Spur zu hinterlassen als die Exemplare, die sich aus der Einziehung durch diesen König gerettet haben. Innerhalb ihrer Gültigkeitsdauer aber kann sie kaum eine andere Wirkung gehabt haben, als dass auch die Goldwährung des Königreichs in Verwirrung ${ }^{1}$ ) ge-

1) Neben den drei Arten von Dnzen, nach denen für gewöhnlich bei Gold gerechnet wurde, der uncia auri, der uncia tarenorum und der uncia augustalium, kommen noch andere Bezeichnungen für die Goldunze vor, von denen es mir zweifelhaft ist, inwiefern sie sich mit jenen decken. Ich entnehme die Beleg. stellen dem ersten Bande meiner Acta imperii. Die uncia auri ad pondus generale, die 1241 (p. 669,7) oder ponderis generalis, die noch unter Karl 1274 (p. 595,2$)$ bei Besoldungen von Beamten in Anwendung kam, dürfte die gewöhnliche im Gewichte von 27,12 Gr. gewesen sein. Aber es werden auch leichtere und schwerere Unzen Gold gegenübergestellt, wie z. B. wenn 1241 oder 1242 gegen einen Beamten Untersuchung verfügt wird, ob er maiori pondere aurum recepit ef minori postea curje solvit (p. 670,25). Es scheint fast, als ob die leichtere in Anwendung kam, wenn die Regierung Zahlungen zu machen, die schwerere aber, wenn sie solche zu empfangen hatte. Wenn eine Pacht an sie 
stürzt wurde, die allerdings etwas anderer Art war als die, der die sicilische Silbermünze durch ihre heillose Verschlechterung unter Kaiser Friedrich II. überliefert wurde.

Zum Schlusse mögen hier um der bequemeren Uebersicht willen die hauptsächlichsten Rechnungsergebnisse der Untersuchung tabellarisch zusammengefasst werden. Von allgemeinerer Bedeutung aber und im Besonderen für die Beurtheilung der Verwaltung Friedrichs II. ist die Erkenntniss nicht ohne Werth, dass er bei der Einführung der Augustalen keine flscalischen Nebenzwecke verfolgt und dass er selbst in den Zeiten grösster Bedrängniss die Goldwährung seines Königreiches unangetastet gelassen hat.

\section{Die Goldmünzen:}

Tarenus

Angustalis

Mittleres Gewicht bei der Prägung . 0,90 Gr. $\quad 5,35$ Gr. davon Feingold . . . . . . 0,61 " 4,57 " Metallwerth überhaupt . . . . $1,72 \mathrm{M}$. $13,22 \mathrm{M}$. Verkehrswerth . . . . . . 1,84 " 13,84 "

II. Die Rechnungsmünze:

Uncia auri (puri) tarenorum angustalium Gewicht . 30 Tari $=27,12 \mathrm{Gr} .30$ Tari $=27,12 \mathrm{Gr} .24$ Tari $=21,69 \mathrm{Gr}$. davon Feingold $\quad 18,37$ Gr. $\quad 18,28$ Gr. Metallwerth $\quad 75,19 \mathrm{M}$. $\quad 51,60 \mathrm{M}$. $\quad 52,88 \mathrm{M}$. Verkehrswerth " $\quad 55,37$, , $\quad 55,36$,

(p. 669,43) ad maius pondus zu entrichten ist, dürfte dies mit der uncia auri ad pondus curie gleich bedeutend sein, nach der 1242 (p. 676,12) der mit Beschlag belegte Kirchenschatz von Girgenti abgeschätzt wurde. Endlich, wenn 1218 (p. 123) eine Schenkung gemacht wird in uncie auri bonorum tarenorum Sicilie ad pondus Baroli, kann daraus doch nur geschlossen werden, dass es ausser den vollwichtigen Tari auch noch schlechtere gab und dass die ersteren damals in Barletta geprägt wurden. - Uebrigens wenn es uns befremdet, dass die uncia augustalium um ein Fünftel leichter war als die uncia tarenorum und statt 30 nur 24 Tari wog, so ist daran zu erinnern, dass nach der Münzerordnung (p. 766,43), die Unze Silber ihrerseits um ein Zehntel schwerer war, als die Unze Gold, also 33 Tari wog.

\section{Verzeichniss der Mutinzabbildungen:}

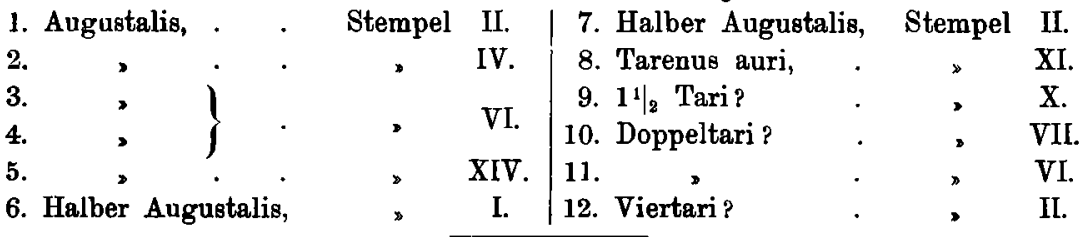

